Article

\title{
Design, Synthesis and Biological Evaluation of Pentacyclic Triterpene Derivatives: Optimization of Anti-ABL Kinase Activity
}

\author{
Halil I. Ciftci ${ }^{1,2}$, Mohamed O. Radwan ${ }^{1,2,3}$, , Safiye E. Ozturk ${ }^{4}$, N. Gokce Ulusoy ${ }^{4}$, Ece Sozer ${ }^{4}$, \\ Doha E. Ellakwa ${ }^{2,5}{ }^{\mathbb{D}}$, Zeynep Ocak ${ }^{6}$, Mustafa Can ${ }^{2,7}$, Taha F.S. Ali ${ }^{2,8}$, Howaida I. Abd-Alla ${ }^{3}{ }^{(D)}$, \\ Nurettin Yayli ${ }^{9}$, Hiroshi Tateishi ${ }^{2}$, Masami Otsuka ${ }^{1,2, *}$ and Mikako Fujita ${ }^{2, *}$ \\ 1 Department of Drug Discovery, Science Farm Ltd., 1-7-30-805 Kuhonji, Chuo-ku, Kumamoto 862-0976, Japan; \\ hiciftci@kumamoto-u.ac.jp (H.I.C.); mohamedradwan@kumamoto-u.ac.jp (M.O.R.) \\ 2 Medicinal and Biological Chemistry Science Farm Joint Research Laboratory, Faculty of Life Sciences, \\ Kumamoto University, 5-1 Oe-honmachi, Chuo-ku, Kumamoto 862-0973, Japan; \\ profdoha@gmail.com (D.E.E.); mustafacan80@yahoo.com (M.C.); tahafaroukali@gmail.com (T.F.S.A.); \\ htateishi@kumamoto-u.ac.jp (H.T.) \\ 3 Chemistry of Natural Compounds Department, Pharmaceutical and Drug Industries Research Division, \\ National Research Centre, Dokki 12622,Cairo, Egypt; howaida_nrc@yahoo.com \\ 4 Chemistry Department, Faculty of Science, Ege University, Erzene Mahallesi, Genclik Caddesi, \\ Bornova/Izmir 35040, Turkey; sfymrt14@gmail.com (S.E.O.); ng.ulusoy@gmail.com (N.G.U.); \\ ecsozer95@gmail.com (E.S.) \\ 5 Department of Biochemistry, Faculty of Pharmacy (Girls), Al-Azhar University, Nasr City 11651, Cairo, Egypt \\ 6 Department of Microbiology, Kocaeli State Hospital, Cedit Mahallesi Gunes Cad, Hastane Yolu Sk, \\ Kocaeli 41300, Turkey; zeynepocak@yahoo.com \\ 7 Department of Engineering Sciences, Faculty of Engineering and Architecture, Izmir Katip Celebi University, \\ Havaalani Sosesi Caddesi No:25, Cigli/Izmir 35620, Turkey \\ 8 Medicinal Chemistry Department, Faculty of Pharmacy, Minia University, Minia 61519, Egypt \\ 9 Faculty of Pharmacy, Karadeniz Technical University, Trabzon 61080, Turkey; yayli@ktu.edu.tr \\ * Correspondence: motsuka@gpo.kumamoto-u.ac.jp (M.O.); mfujita@kumamoto-u.ac.jp (M.F.); \\ Tel.: +81-96-371-4622 (M.F.)
}

Academic Editors: Nazim Sekeroglu, Anake Kijjoa and Sevgi Gezici Received: 26 July 2019; Accepted: 26 September 2019; Published: 30 September 2019

Abstract: Imatinib, an Abelson (ABL) tyrosine kinase inhibitor, is a lead molecular-targeted drug against chronic myelogenous leukemia (CML). To overcome its resistance and adverse effects, new inhibitors of ABL kinase are needed. Our previous study showed that the benzyl ester of gypsogenin (1c), a pentacyclic triterpene, has anti-ABL kinase and a subsequent anti-CML activity. To optimize its activities, benzyl esters of carefully selected triterpenes (PT1-PT6), from different classes comprising oleanane, ursane and lupane, and new substituted benzyl esters of gypsogenin (GP1-GP5) were synthesized. All of the synthesized compounds were purified and charachterized by different spectroscopic methods. Cytotoxicity of the parent triterpenes and the synthesized compounds against CML cell line K562 was examined; revealing three promising compounds PT5, GP2 and GP5 (IC 50 5.46, 4.78 and $3.19 \mu \mathrm{M}$, respectively). These compounds were shown to inhibit extracellular signal-regulated kinase (ERK) downstream signaling, and induce apoptosis in K562 cells. Among them, PT5 was identified to have in vitro activity $\left(\mathrm{IC}_{50}=1.44 \mu \mathrm{M}\right)$ against ABL1 kinase, about sixfold of 1c, which was justified by molecular docking. The in vitro activities of GP2 and GP5 are less than PT5, hence they were supposed to possess other more mechanisms of cytotoxicity. In general, our design and derivatizations resulted in enhancing the activity against ABL1 kinase and CML cells.

Keywords: leukemia; chronic myelogenous leukemia; ABL kinase; pentacyclic triterpenes; gypsogenin; apoptosis 


\section{Introduction}

Leukemia, a group of blood cancer which initiates in the bone marrow resulting in an abnormal production of white blood cells, is the leading cause of cancer-related deaths in a wide population range, from children to aged people. In 2016, the annual number of new cases of leukemia was 467,000 worldwide, with 310,000 leukemia deaths [1]. Based on disease progression and cell types, leukemia is classified into four main types, acute lymphocytic leukemia (ALL), chronic lymphocytic leukemia (CLL), acute myelogenous leukemia (AML) and chronic myelogenous leukemia (CML) [2].

CML is a slow-growing type of leukemia characterized by a chromosome translocation between chromosome 9 and 22 to form the Philadelphia ( $\mathrm{Ph}$ ) chromosome [3-6]. The Ph chromosome contains the connected breakpoint cluster region (BCR) and the Abelson (ABL) oncogene, resulting in BCR-ABL fusing gene with constitutive tyrosine kinase activity [6-9]. The tyrosine kinase protein plays an important role in the activation of signalling pathways (Janus kinase: JAK, signal transducers and activators of transcription: STAT, Src family kinase: SFK, phosphoinositide 3-kinase: PI3K, protein kinase B: AKT and extracellular signal-regulated kinase: ERK) and cellular processes such as growth, differentiation, and metabolism [10-12]. The introduction of imatinib mesylate, a representative molecular-targeted drug and the first tyrosine kinase inhibitor (TKI) with specific activity against $\mathrm{ABL}$, controls CML in chronic phase. However, a significant percentage of CML patients treated with imatinib developed drug resistance and adverse side effects [13-15]. Second and third generation of TKIs such as dasatinib, nilotinib and ponatinib have been developed to overcome these major limitations, but patients treated with these TKIs still did not achieve desired CML treatment and some patients were left with unexpected lung and vascular problems [16-19]. Hence, there is an urgent need for new therapeutic agents for CML patients.

In recent years, natural products and their derivatives have attracted great deal of interest as a source for the development of new diverse bioactive compounds due to their high safety profile and cost effectiveness [20-26]. Among them, pentacyclic triterpenes are the subjects of numerous studies carried out by chemists engaged in organic synthesis and pharmacology. A large number of pentacyclic triterpenes such as glycyrrhetic acid, oleanolic acid, ursolic acid, betulinic acid and their derivatives have been reported to exhibit various biological activities including antiinflammatory, antimicrobial, antiviral, antioxidant, antitumor and anticancer [27-32]. However, a pentacyclic triterpene named gypsogenin has not yet received significant attention in drug discovery. Gypsogenin extracted from Gypsophila with sugar chains is a unique pentacyclic triterpene possessing an aldehyde group at its C4 distinguished from other pentacyclic triterpenes. In our previous studies [33,34] and [35], it was demonstrated that gypsogenin and its derivatives exhibit remarkable activities against diverse human cancer cell lines. Furthermore, we recently discovered that the benzyl ester of gypsogenin compound 1c has anti-ABL kinase and anti-CML activities [36]. To the best of our knowledge, this was the first study to show the inhibitory activity of pentacyclic triterpenoids and their derivatives on ABL kinase. In this study, we conduct biological evaluation of some pentacyclic triterpenes and their derivatives including newly synthesized compounds to optimize anti-ABL kinase and anti-CML activities.

\section{Results and Discussion}

As representative pentacyclic triterpenes, we selected six compounds, asiatic acid (AA), betulinic acid (BA), glycyrrhetinic acid (GA), hederagenin (HE), oleanolic acid (OA) and ursolic acid (UA) (Figure 1). AA and UA represent the ursane type, and BA represents the lupane type. GA, HE and OA are of the oleanane type. On these pentacyclic triterpenes and each benzyl ester like 1c (PT1-PT6), biological activities were examined. Furthermore, five new substituted benzyl esters of gypsogenin GP1-GP5 were also designed. GP1 has a 4-methoxy substitution as an electron-donating group whereas other compounds have electron-withdrawing substations such as fluoro-, trifluoromethyl-, and cyano- groups. 


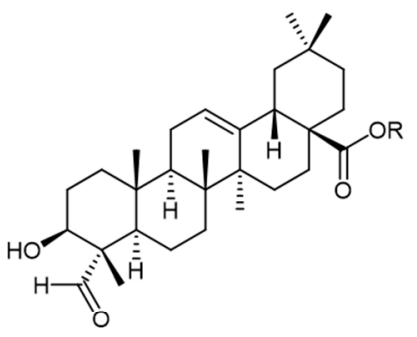

GP (Gypsogenin) R = H

1c $\mathrm{R}=$ benzyl

GP1 R = p-(methoxy)benzyl

GP2 R = 3,5-bis(trifluoromethyl)benzyl

GP3 R = p-(trifluoromethyl)benzyl

GP4 R = 3,5-difluorobenzyl

GP5 R = p-cyanobenzyl

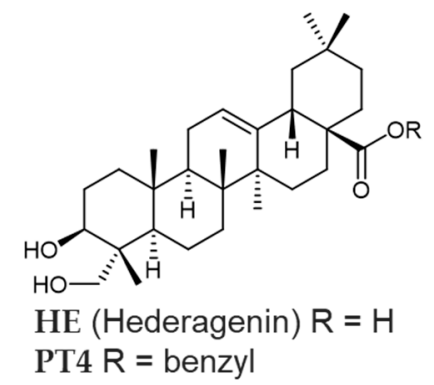

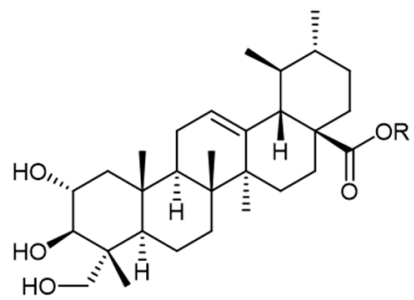

AA (Asiatic acid) $\mathrm{R}=\mathrm{H}$ PT1 R = benzyl

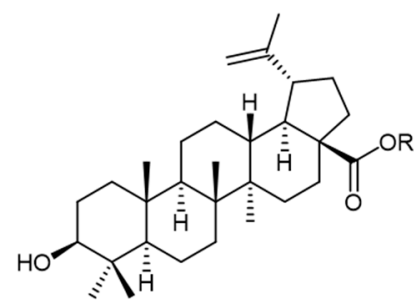

BA (Betulinic acid) $\mathrm{R}=\mathrm{H}$ PT2 R = benzyl

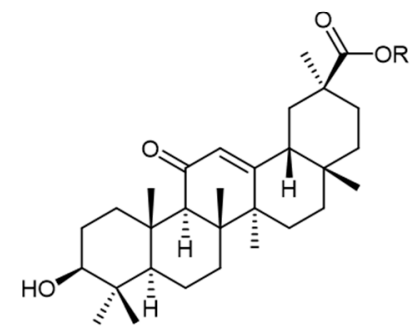

GA (Glycyrrhetinic acid) R = H

PT3 R = benzyl

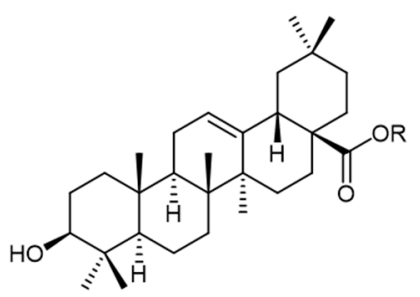

OA (Oleanolic acid) $\mathrm{R}=\mathrm{H}$ PT5 R = benzyl

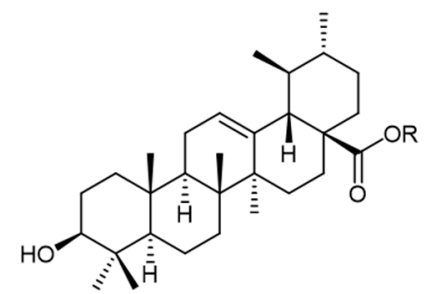

UA (Ursolic acid) $\mathrm{R}=\mathrm{H}$ PT6 R = benzyl

Figure 1. Structures of pentacyclic triterpenes and its derivatives used in this study.

The synthesis of benzyl esters of different triterpenes PT1-PT6 and the new substituted benzyl esters of gypsogenin GP1-GP5 was performed as outlined in Scheme 1. AA, BA, GA, HE, OA and UA were reacted with benzyl bromide in the presence of potassium carbonate as a base to afford the corresponding esters PT1, PT2, PT3, PT4, PT5, and PT6 respectively in good yields (44-81\%). In a similar way, GP was reacted with different substituted benzyl bromides producing five new compounds GP1, GP2, GP3, GP4, and GP5 in 40-48\% yield.

To evaluate the antiproliferative effects of all compounds against CML cell line K562, MTT assay was carried out. The results were summarized in Table 1 . Imatinib was used as a control. $\mathrm{IC}_{50}$ values of free triterpenes were detected to be higher than $10 \mu \mathrm{M}$. Introduction of benzyl ester group into triterpene led to an increase in anticancer activity on K562 except for PT3 and PT6, based on GA and UA, respectively. Specially, PT4 has more than 15 times stronger cytotoxicity than its parent triterpene HE. The $\mathrm{IC}_{50}$ values of all new gypsogenin derivatives GP1-GP5 were lower than $6 \mu \mathrm{M}$. In order to investigate whether PT1-PT6 and GP1-GP5 are toxic to healthy blood cells or not, the cytotoxic effects of these compounds on peripheral blood mononuclear cells (PBMC) were also examined. Each compound, except HE which lacks cytotoxicity against K562, showed a considerable selectivity index (SI). Notably, free triterpenes GP and OA have $\mathrm{IC}_{50}$ of around $13 \mu \mathrm{M}$ against $\mathrm{K} 562$ and SI of more than 7. We identified GP2, GP5 and PT5 as the strongest and selective anticancer agents on CML cell line. These three compounds were also more selective than imatinib. 


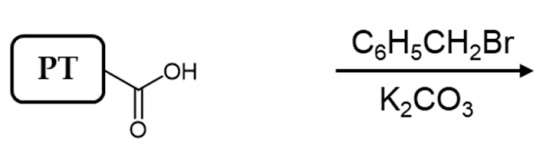

AA, BA, GA, HE, OA, UA<smiles>O=C(OCc1ccccc1)C12CCC(CC1)C2</smiles>

PT1, PT2, PT3, PT4, PT5, PT6

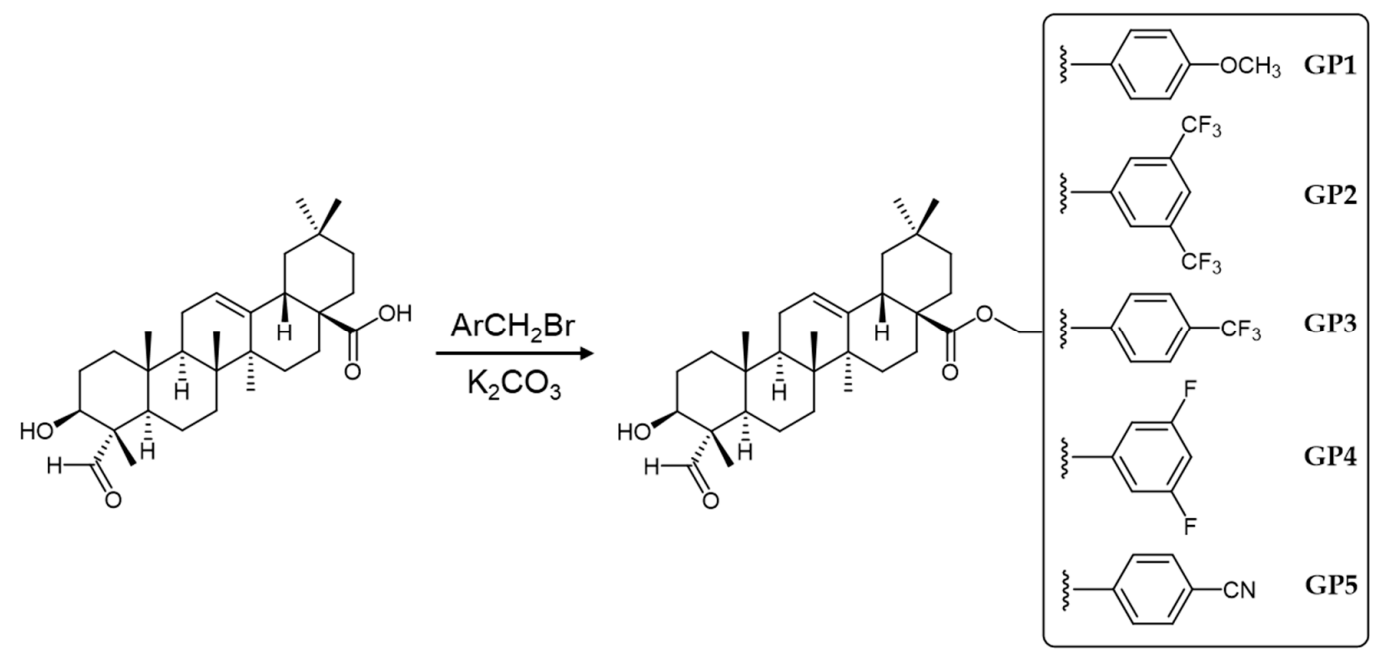

Scheme 1. Synthesis of PT1-PT6 and GP1-GP5. PT: pentacyclic triterpene skeleton.

Table 1. The cytotoxic effects of all compounds on K562 and peripheral blood mononuclear cells (PBMC).

\begin{tabular}{|c|c|c|c|c|}
\hline & \multirow{2}{*}{ ID } & \multicolumn{2}{|c|}{$\mathrm{IC}_{50}$ Values $(\mu \mathrm{M})^{\mathrm{a}}$} & \multirow{2}{*}{$\mathbf{S I}^{\mathbf{b}}$} \\
\hline & & K562 Cell Line & PBMC & \\
\hline \multirow{7}{*}{ Free Triterpenes } & GP & $12.67 \pm 2.15$ & $>100$ & $>7.89$ \\
\hline & AA & $29.31 \pm 3.42$ & $>100$ & $>3.41$ \\
\hline & BA & $52.27 \pm 4.79$ & $>100$ & $>1.91$ \\
\hline & GA & $41.03 \pm 5.34$ & $>100$ & $>2.43$ \\
\hline & $\mathrm{HE}$ & $>100$ & $>100$ & $>1$ \\
\hline & $\mathbf{O A}$ & $13.46 \pm 2.55$ & $>100$ & $>7.43$ \\
\hline & UA & $16.51 \pm 1.13$ & $>100$ & $>6.06$ \\
\hline \multirow{7}{*}{ Triterpenes benzyl esters } & 1c & $8.94 \pm 1.73$ & $>100$ & $>11.19$ \\
\hline & PT1 & $4.10 \pm 0.85$ & $15.54 \pm 1.08$ & 3.79 \\
\hline & PT2 & $8.49 \pm 1.22$ & $27.96 \pm 2.74$ & 3.29 \\
\hline & PT3 & $69.50 \pm 8.43$ & $>100$ & $>1.44$ \\
\hline & PT4 & $6.48 \pm 1.27$ & $23.08 \pm 3.11$ & 3.56 \\
\hline & PT5 & $5.46 \pm 1.51$ & $50.21 \pm 4.58$ & 9.19 \\
\hline & PT6 & $21.77 \pm 1.24$ & $43.31 \pm 1.93$ & 1.99 \\
\hline \multirow{5}{*}{$\begin{array}{l}\text { Gypsogenin substituted- } \\
\text { benzyl esters }\end{array}$} & GP1 & $5.53 \pm 1.78$ & $25.05 \pm 2.92$ & 4.53 \\
\hline & GP2 & $4.78 \pm 1.25$ & $52.76 \pm 3.82$ & 11.04 \\
\hline & GP3 & $4.58 \pm 1.49$ & $23.22 \pm 3.08$ & 5.07 \\
\hline & GP4 & $5.13 \pm 1.38$ & $28.28 \pm 2.25$ & 5.51 \\
\hline & GP5 & $3.19 \pm 1.21$ & $25.60 \pm 2.18$ & 8.03 \\
\hline Control & Imatinib $^{\mathrm{c}}$ & $5.49 \pm 1.81$ & $28.66 \pm 3.16$ & 5.22 \\
\hline
\end{tabular}

The cytotoxic effects of these compounds were also evaluated on different leukemic cells (HL-60, MT-2 and Jurkat), HeLa, MCF7 and A549 cell lines as shown in Table 2. Among them, the cyano-substituted compound GP5 were found as the most effective on all cancer cell lines. GP2 
and PT5 were inactive against A549 cell line at $100 \mu \mathrm{M}$ concentration. PT5 was less active compared to GP2 and GP5 on HL-60, Jurkat, HeLa and MCF7 cell lines. PT5 exhibited selectivity against leukemia cells like imatinib.

Due to their significant anticancer potentials on CML, GP2, GP5 and PT5 were further investigated for their apoptotic activities in K562 cells using Hoechst 33342/annexin V/ethidium homodimer III staining method. K562 cells were treated with GP2, GP5 and PT5 at their $\mathrm{IC}_{50}$ concentrations for 8 $\mathrm{h}$, then stained and observed by a florescence microscope (Figure 2a). The apoptotic effects of GP2, GP5, PT5 and imatinib on CML cell line were determined as 24\%, 9.5\%, 14\% and 18\%, respectively, while their late apoptotic/necrotic effects were determined as $4.5 \%, 5.1 \%, 11 \%$ and 5.6\% respectively (Figure 2b). According to these findings, GP2 having 3,5-ditrifluoromethyl substitutions induced more pronounced apoptosis of K562 cells than GP5, PT5 and imatinib.

(a)

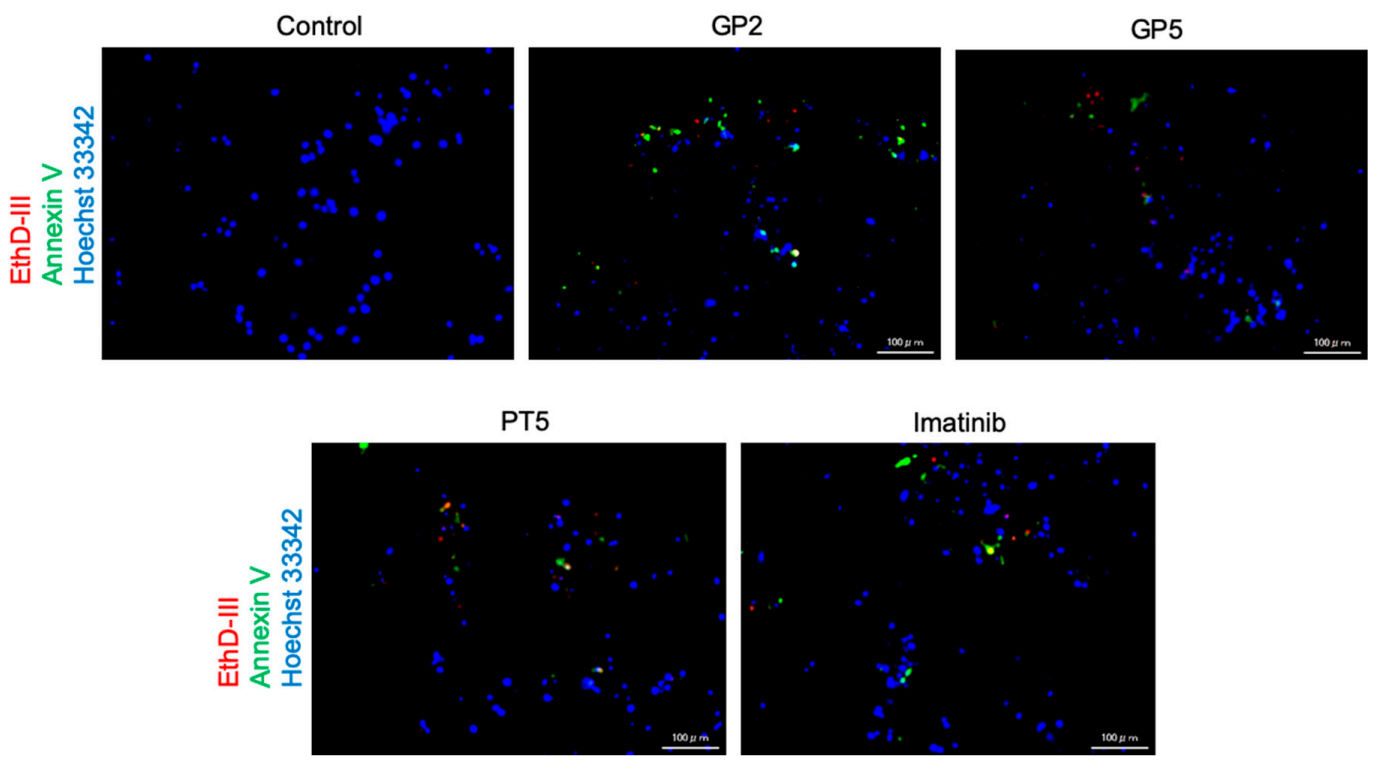

(b)

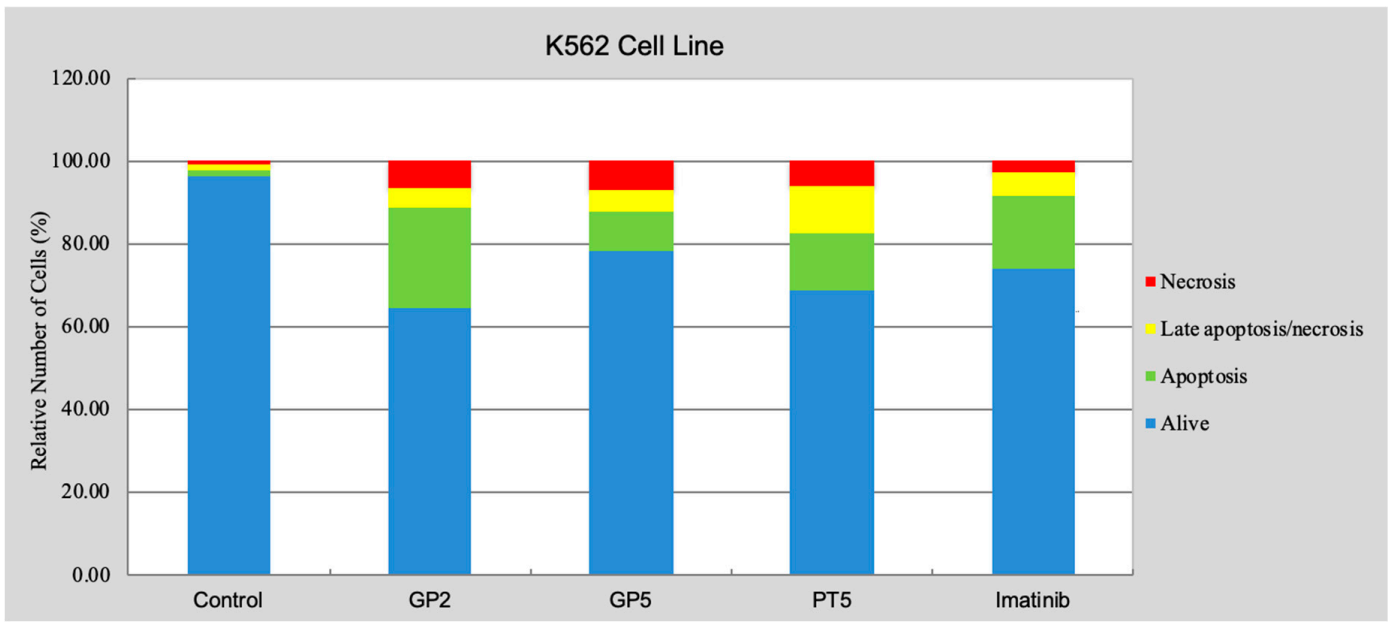

Figure 2. Alteration in $\mathrm{K} 562$ cells at $\mathrm{IC}_{50}$ concentrations of control, GP2, GP5, PT5 and imatinib for 8 h. (a) Fluorescent microscopic images. (b) A total of approximately 100 stained cells was selected randomly in each experiment of (a) and were classified into 4 types "alive" (blue), "apoptosis" (green), "necrosis or late apoptosis" (both green and red, yellow) and "necrosis" (red). 
Table 2. The cytotoxicity of selected compounds against other cancer cell lines than K562.

\begin{tabular}{ccccccc}
\hline & \multicolumn{7}{c}{ IC 50 Values $(\mu \mathrm{M})$} \\
\cline { 2 - 7 } & HL-60 & MT-2 & Jurkat & HeLa & MCF7 & A549 \\
\hline GP2 & $5.27 \pm 0.42$ & $36.82 \pm 4.21$ & $9.14 \pm 0.71$ & $35.28 \pm 2.84$ & $51.58 \pm 4.75$ & $>100$ \\
\hline GP5 & $4.36 \pm 0.28$ & $7.26 \pm 0.97$ & $4.83 \pm 0.35$ & $5.66 \pm 0.32$ & $15.31 \pm 2.11$ & $24.55 \pm 1.94$ \\
\hline PT5 & $15.71 \pm 1.36$ & $34.70 \pm 3.85$ & $13.85 \pm 1.58$ & $67.05 \pm 4.19$ & $53.91 \pm 6.46$ & $>100$ \\
\hline Imatinib & $11.75 \pm 1.08$ & $12.96 \pm 1.33$ & $9.69 \pm 0.63$ & $28.68 \pm 1.92$ & $90.56 \pm 5.81$ & $95.07 \pm 8.33$ \\
\hline
\end{tabular}

As a consequence of the central role of ABL kinase in cellular processes including cell replication and apoptosis, GP2, GP5 and PT5 were selected for further investigation of the potential inhibitory activity against ABL kinase using multipoint dose-response experiments (Table 3). Among them, PT5 was found to be the most potent $\mathrm{ABL}$ kinase inhibitor with the $\mathrm{IC}_{50}$ value of $1.44 \mu \mathrm{M}$, followed by GP5 and GP2 with $\mathrm{IC}_{50}$ values of $6.16 \mu \mathrm{M}$ and $7.19 \mu \mathrm{M}$, respectively. $\mathrm{IC}_{50}$ of PT5 is six times stronger than 1c. Although it is still incomparable to imatinib $\left(\mathrm{IC}_{50} 0.21 \mu \mathrm{M}\right)$, they showed similar cellular activity ( $\mathrm{IC}_{50} \sim 5.5 \mu \mathrm{M}$ ) against $\mathrm{K} 562$ cells (Table 1 ) and similar selectivity against various cancer cell lines (Table 2). PT5 is considered to express more efficient cellular activity, but the detailed reason remains elusive.

Table 3. The Abelson (ABL) 1 tyrosine kinase inhibition of selected compounds and imatinib.

\begin{tabular}{cccccc}
\hline & \multicolumn{5}{c}{ IC $_{50}$ Values $(\boldsymbol{\mu M})$} \\
\cline { 2 - 6 } & 1c $^{\mathbf{a}}$ & GP2 & GP5 & PT5 & Imatinib \\
\hline ABL1 & $8.71 \pm 1.14$ & $7.19 \pm 0.65$ & $6.16 \pm 0.63$ & $\mathbf{1 . 4 4} \pm \mathbf{0 . 1 2}$ & $0.21 \pm 0.44$ \\
\hline \multicolumn{5}{c}{ a A value in the previous paper [36]. }
\end{tabular}

To investigate this outstanding activity of PT5, we conducted a molecular docking study into the ATP-binding site of ABL kinase. Herein, we used the most updated version of Molecular Operating Environment (MOE) software that explains the slightly shifted placement of $\mathbf{1 c}$ from our previous work [36]. Both compounds demonstrated a similar and superimposed binding mode typically settling three $\mathrm{CH}-\pi$ interaction with His361, Asp381 and Phe401. Their hydrophobic fused cyclohexyl rings lie in proximity to hydrophobic residues Val289, Phe359 and Ile360, Ala380 and Phe382 (Figure 3). We believe that the presence of the polar aldehyde group on C4 of compound 1c (binding energy -7.11) in proximity to the hydrophobic residues Ala399 and Phe401 is unfavorable compared to the lower methyl group of PT5 which is at the same position (binding energy -7.31). Finally, the rigid skeleton of PT5 limits its deep burial into the pocket as fulfilled by imatinib.

In order to explore the inhibitory potency of GP2, GP5, and PT5 on downstream signaling of BCR-ABL kinase such as rapidly accelerated fibrosarcoma (Raf)/mitogen-activated protein kinase/extracellular signal-regulated kinase (MEK)/extracellular signal-regulated kinase (ERK), ERK1/2 and their phosphorylated forms in K562-treated with GP2, GP5, or PT5 were examined at $10 \mu \mathrm{M}$ concentration for $6 \mathrm{~h}$ (Figure 4). In the presence of the titled compounds, amount of ERK1/2 moderately decreased. In contrast, the reduction of phosphorylated ERK1/2 was prominent where the three compounds exhibited inhibitory effect of ERK phosphorylation in a similar pattern to imatinib. The inhibitory activity against ERK phoshorylation is not in accordance with cytotoxicity data in K562 cells (Table 1). This suggests that there is a pathway other than ERK signaling suppressed by the titled compounds. 


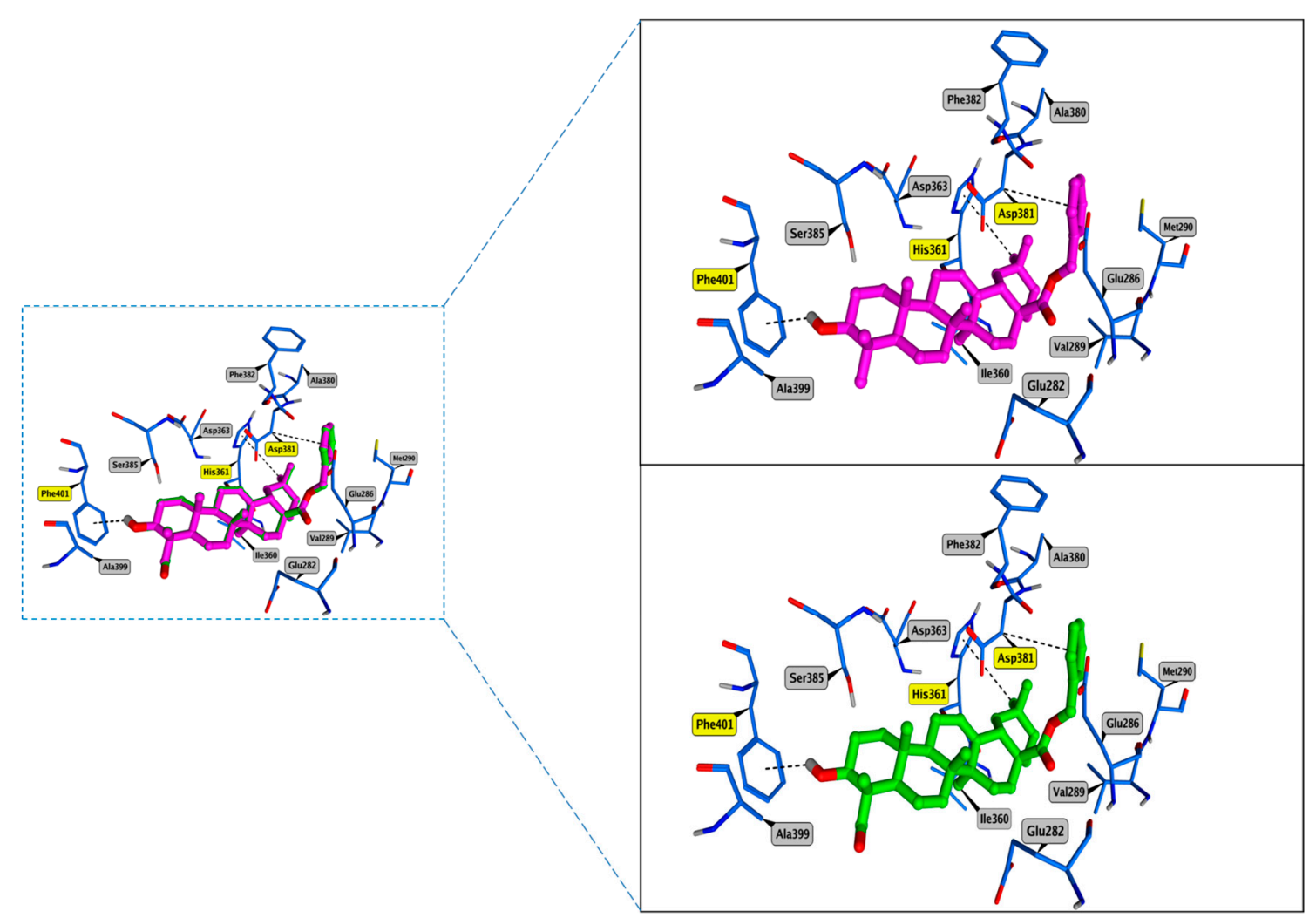

Figure 3. Superimposition of PT5 (magenta) and 1c (green) into ATP binding site of ABL kinase (PDB: 1IEP) as predicted by MOE 2019.0101 followed by a zoom-in separate view for each compound. Amino acids are depicted in blue sticks. All heteroatoms are colored by element. Ligand-protein interactions are shown as black-dash lines. Some amino acid residues are hidden for clarity purpose.

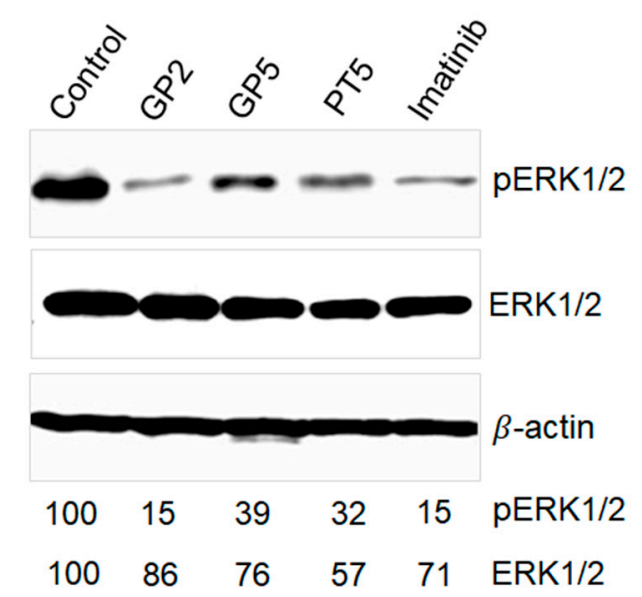

Figure 4. The effect of GP2, GP5, PT5 and imatinib on ERK signaling. K562 cells were incubated with the compounds at $10 \mu \mathrm{M}$ concentration for $6 \mathrm{~h}$, and then immunoblot analysis was conducted. Band intensity was quantitated and relative values are shown below.

\section{Materials and Methods}

\subsection{Chemistry}

All reactions were performed in an efficient fume hood. Chemicals were purchased from Sigma-Aldrich (St. Louis, MO, USA), Honeywell Fluka (Morristown, NJ, USA), Kanto Chemical (Tokyo, Japan), Nacalai Tesque (Kyoto, Japan), Tokyo Chemical Industry (Tokyo, Japan) and FUJIFILM Wako (Osaka, Japan). Commercially available reagent-grade chemicals were used without further 
purification. The reaction progress was monitored by a thin layer chromatography (TLC) on precoated plates [Merck (Darmstadt, Germany) TLC sheets silica 60 F254]. The flash column chromatography was carried out on Silica Gel 60N [40-100 mesh, Kanto Chemical (Tokyo, Japan)]. Melting points were determined on a Yanaco (Kyoto, Japan) melting point apparatus and were uncorrected. ${ }^{1} \mathrm{H}$ and ${ }^{13} \mathrm{C}$ NMR spectra were registered on a Bruker (Billerica, MA, USA) Avance 600 (600 MHz). Chemical shits are referenced to TMS. Mass spectra (MS) and high-resolution mass spectra (HRMS) were recorded on a JEOL (Tokyo, Japan) JMS-DX303HF using positive fast atom bombardment (FAB) with 3-nitrobenzyl alcohol as the matrix.

\subsection{General Method of Preparation of PT1-PT5 and GP1-GP5 Compounds}

A $0.5 \mathrm{mmol}$ of a triterpenic compound was dissolved in $5 \mathrm{~mL}$ dry DMF followed by the addition of anhydrous $\mathrm{K}_{2} \mathrm{CO}_{3}(1.5 \mathrm{mmol})$ and stirred for $30 \mathrm{~min}$. The appropriate free or substituted benzyl bromide was added and the mixture was stirred for an additional $6 \mathrm{~h}$ at room temperature. Upon the consumption of the reactant, $5 \mathrm{~mL}$ of the brine solution was added and the mixture was extracted with ethyl acetate. The organic phase was washed with water until $\mathrm{pH}=7$, dried over anhydrous sodium sulfate, filtered and concentrated under vacuo. The resulted residue was applied for column chromatography using an appropriate eluent [31].

Asiatic acid benzyl ester (PT1) [37]. Compound PT1 was obtained from AA and benzyl bromide as a white solid $(173 \mathrm{mg}, 60 \%) .{ }^{1} \mathrm{H}$ NMR $\left(600 \mathrm{MHz}, \mathrm{CDCl}_{3}\right) 8: 7.36-7.30(\mathrm{~m}, 5 \mathrm{H}, \mathrm{H}-\mathrm{Ar}), 5.23(\mathrm{t}, J=3.6 \mathrm{~Hz}$, $1 \mathrm{H}), 5.09\left(\mathrm{~d}, J=12.5 \mathrm{~Hz}, 1 \mathrm{H}, \mathrm{Bn}-\mathrm{CH} H^{\prime}\right), 4.98\left(\mathrm{~d}, J=12.5 \mathrm{~Hz}, 1 \mathrm{H}, \mathrm{Bn}-\mathrm{CHH}^{\prime}\right), 3.77-3.73(\mathrm{~m}, 1 \mathrm{H}), 3.64$ $(\mathrm{d}, J=10.6 \mathrm{~Hz}, 1 \mathrm{H}), 3.51(\mathrm{brs}, 1 \mathrm{H}), 3.41(\mathrm{~d}, J=9.8 \mathrm{~Hz}, 2 \mathrm{H}), 2.64(\mathrm{brs}, 2 \mathrm{H}), 2.26(\mathrm{~d}, J=11.3 \mathrm{~Hz}, 1 \mathrm{H})$, 2.01-1.86 (m, 4H), 1.80-1.67 (m, 5H), 1.63-1.55 (m, 2H), 1.50-1.45 (m, 2H), 1.36-1.25 (m, 5H), 1.07 (s, $3 \mathrm{H}), 1.05-1.02(\mathrm{~m}, 2 \mathrm{H}), 1.00(\mathrm{~s}, 3 \mathrm{H}), 0.93(\mathrm{~d}, J=6.5 \mathrm{~Hz}, 3 \mathrm{H}), 0.86(\mathrm{~s}, 3 \mathrm{H}), 0.85(\mathrm{~d}, J=6.5 \mathrm{~Hz}, 3 \mathrm{H}), 0.63(\mathrm{~s}$, $3 \mathrm{H}) .{ }^{13} \mathrm{C}$ NMR $\left(150 \mathrm{MHz}, \mathrm{CDCl}_{3}\right) \delta: 177.29,138.22,136.36,128.42,128.16,127.97,125.41,80.56,70.62$, 68.77, 66.01, 52.87, 49.15, 48.12, 47.48, 46.29, 42.44, 42.14, 39.56, 39.11, 38.84, 38.15, 36.62, 32.68, 30.66, 27.92, 24.23, 23.64, 23.33, 21.17, 18.33, 17.16, 17.05, 17.01, 12.79. MS (FAB) $m / z 601.2(\mathrm{M}+\mathrm{Na})^{+}$; HRMS (FAB). Calcd for $\mathrm{C}_{37} \mathrm{H}_{54} \mathrm{O}_{5} \mathrm{Na:}$ 601.3869. Found: 601.3868. (Figure S1).

Betulinic acid benzyl ester (PT2) [38]. Compound PT2 was obtained from BA and benzyl bromide as a white solid $(136 \mathrm{mg}, 50 \%) .{ }^{1} \mathrm{H}$ NMR $\left(600 \mathrm{MHz}, \mathrm{CDCl}_{3}\right) \delta: 7.35-7.29(\mathrm{~m}, 5 \mathrm{H}, \mathrm{H}-\mathrm{Ar}), 5.12(\mathrm{~d}$, $J=12.3 \mathrm{~Hz}, 1 \mathrm{H}, \mathrm{Bn}-\mathrm{CHH}), 5.07\left(\mathrm{~d}, J=12.3 \mathrm{~Hz}, 1 \mathrm{H}, \mathrm{Bn}-\mathrm{CHH}^{\prime}\right), 4.70(\mathrm{~d}, J=2.3 \mathrm{~Hz}, 1 \mathrm{H}), 4.57(\mathrm{dd}, J=2.3$, $1.4 \mathrm{~Hz}, 1 \mathrm{H}), 3.17-3.13(\mathrm{~m}, 1 \mathrm{H}), 3.02-2.98(\mathrm{~m}, 1 \mathrm{H}), 2.27-2.24(\mathrm{~m}, 1 \mathrm{H}), 2.18-2.14(\mathrm{~m}, 1 \mathrm{H}), 1.89-1.83(\mathrm{~m}$, $2 \mathrm{H}), 1.63-1.56(\mathrm{~m}, 3 \mathrm{H}), 1.54(\mathrm{~s}, 3 \mathrm{H}), 1.52-1.16(\mathrm{~m}, 14 \mathrm{H}), 1.08(\mathrm{dt}, J=13.5,3.1 \mathrm{~Hz}, 1 \mathrm{H}), 0.99(\mathrm{td}, J=12.8$, $4.6 \mathrm{~Hz}, 1 \mathrm{H}), 0.93(\mathrm{~s}, 3 \mathrm{H}), 0.92(\mathrm{~s}, 3 \mathrm{H}), 0.86(\mathrm{td}, J=12.8,3.9 \mathrm{~Hz}, 1 \mathrm{H}), 0.78(\mathrm{~s}, 3 \mathrm{H}), 0.74(\mathrm{~s}, 3 \mathrm{H}), 0.73(\mathrm{~s}$, $3 \mathrm{H}), 0.65-0.63(\mathrm{~m}, 1 \mathrm{H}) .{ }^{13} \mathrm{C} \mathrm{NMR}\left(150 \mathrm{MHz}, \mathrm{CDCl}_{3}\right) \delta 175.82,150.59,136.52,128.50,128.25,128.06$, $109.57,79.01,65.74,56.58,55.39,50.60,49.50,46.96,42.42,40.69,38.88,38.75,38.24,37.21,36.96,34.34$, $32.15,30.62,29.60,28.00,27.45,25.58,20.91,19.39,18.32,16.13,15.86,15.36,14.69$. MS (FAB) $m / z 569.5$ $(\mathrm{M}+\mathrm{Na})^{+}$; HRMS (FAB). Calcd for $\mathrm{C}_{37} \mathrm{H}_{54} \mathrm{O}_{3} \mathrm{Na}$ : 569.3971. Found: 569.4046. (Figure S2).

Glycyrrhetic acid benzyl ester (PT3) [31,38]. Compound PT3 was obtained from GA and benzyl bromide as a white solid (123 mg, 44\%). ${ }^{1} \mathrm{H}$ NMR $\left(600 \mathrm{MHz}, \mathrm{CDCl}_{3}\right) \delta: 7.40-7.32(\mathrm{~m}, 5 \mathrm{H}, \mathrm{H}-\mathrm{Ar}), 5.55(\mathrm{~s}$, 1H), $5.20\left(\mathrm{~d}, J=12.2 \mathrm{~Hz}, 1 \mathrm{H}, \mathrm{Bn}-\mathrm{CHH}^{\prime}\right), 5.09\left(\mathrm{~d}, J=12.2 \mathrm{~Hz}, 1 \mathrm{H}, \mathrm{Bn}-\mathrm{CHH}^{\prime}\right), 3.24-3.21(\mathrm{~m}, 1 \mathrm{H}), 2.79$ $(\mathrm{dt}, J=13.4,3.5 \mathrm{~Hz}, 1 \mathrm{H}), 2.32(\mathrm{~s}, 1 \mathrm{H}), 2.05-2.01(\mathrm{~m}, 3 \mathrm{H}), 1.93(\mathrm{ddd}, J=13.6,4.3,2.7 \mathrm{~Hz}, 1 \mathrm{H}), 1.81(\mathrm{td}$, $J=13.6,4.3 \mathrm{~Hz}, 1 \mathrm{H}), 1.67-1.61(\mathrm{~m}, 4 \mathrm{H}), 1.59-1.57(\mathrm{~m}, 1 \mathrm{H}), 1.47-1.42(\mathrm{~m}, 1 \mathrm{H}), 1.39(\mathrm{ddd}, J=11.0,6.9$, $2.7 \mathrm{~Hz}, 2 \mathrm{H}), 1.35(\mathrm{~s}, 3 \mathrm{H}), 1.32-1.26(\mathrm{~m}, 4 \mathrm{H}), 1.16(\mathrm{~s}, 3 \mathrm{H}), 1.13(\mathrm{~s}, 3 \mathrm{H}), 1.11(\mathrm{~s}, 3 \mathrm{H}), 1.00(\mathrm{~s}, 3 \mathrm{H}), 0.99-0.94$ $(\mathrm{m}, 1 \mathrm{H}), 0.80(\mathrm{~s}, 3 \mathrm{H}), 0.73(\mathrm{~s}, 3 \mathrm{H}), 0.69(\mathrm{dd}, J=11.9,1.5 \mathrm{~Hz}, 1 \mathrm{H}) .{ }^{13} \mathrm{C} \mathrm{NMR}\left(150 \mathrm{MHz}, \mathrm{CDCl}_{3}\right) \delta: 200.11$, 176.21, 168.97, 136.16, 128.63, 128.56, 128.31, 128.26, 78.80, 66.24, 61.82, 54.98, 48.23, 45.37, 44.00, 43.20, $41.12,39.18,39.15,37.68,37.11,32.80,31.80,31.20,28.43,28.30,28.12,27.34,26.50,26.44,23.37,18.70$, $17.52,16.38,15.58$. MS (FAB) $m / z 561.5(\mathrm{M}+\mathrm{H})^{+}$; HRMS (FAB). Calcd for $\mathrm{C}_{37} \mathrm{H}_{53} \mathrm{O}_{4}$ : 561.3944. Found: 561.3970. (Figure S3).

Hederagenin benzyl ester (PT4) [39]. Compound PT4 was obtained from HE and benzyl bromide as a white solid (160 mg, 57\%). ${ }^{1} \mathrm{H}$ NMR $\left(600 \mathrm{MHz}, \mathrm{CDCl}_{3}\right)$ 8: 7.36-7.29 (m, 5H, H-Ar), $5.28(\mathrm{t}, J=3.6 \mathrm{~Hz}$, 
$1 \mathrm{H}), 5.09\left(\mathrm{~d}, J=12.6 \mathrm{~Hz}, 1 \mathrm{H}, \mathrm{Bn}-\mathrm{CHH}^{\prime}\right), 5.04\left(\mathrm{~d}, J=12.6 \mathrm{~Hz}, 1 \mathrm{H}, \mathrm{Bn}-\mathrm{CHH}^{\prime}\right), 3.72(\mathrm{~d}, J=10.3 \mathrm{~Hz}, 1 \mathrm{H})$, 3.64-3.62 (m, 1H), $3.42(\mathrm{~d}, J=10.3 \mathrm{~Hz}, 1 \mathrm{H}), 2.90(\mathrm{dd}, J=13.7,4.3 \mathrm{~Hz}, 1 \mathrm{H}), 2.55(\mathrm{brs}, 1 \mathrm{H}), 2.34(\mathrm{brs}, 1 \mathrm{H})$, $1.97(\mathrm{td}, J=13.9,4.4 \mathrm{~Hz}, 1 \mathrm{H}), 1.85(\mathrm{dd}, J=8.9,3.6 \mathrm{~Hz}, 2 \mathrm{H}), 1.71(\mathrm{td}, J=13.9,4.4 \mathrm{~Hz}, 1 \mathrm{H}), 1.67-1.62$ (m, 3H), 1.57-1.51 (m, 2H), 1.40-1.33 (m, 3H), 1.42-1.33 (m, 4H), 1.23-1.13 (m, 3H), $1.12(\mathrm{~s}, 3 \mathrm{H}), 1.03$ $(\mathrm{dt}, J=13.5,3.1 \mathrm{~Hz}, 1 \mathrm{H}), 0.98-0.94(\mathrm{~m}, 1 \mathrm{H}), 0.93(\mathrm{~s}, 3 \mathrm{H}), 0.92(\mathrm{~s}, 3 \mathrm{H}), 0.89(\mathrm{~s}, 3 \mathrm{H}), 0.89(\mathrm{~s}, 3 \mathrm{H}), 0.84(\mathrm{dd}$, $J=10.6,3.1 \mathrm{~Hz}, 1 \mathrm{H}), 0.60(\mathrm{~s}, 3 \mathrm{H}) .{ }^{13} \mathrm{C} \mathrm{NMR}\left(150 \mathrm{MHz}, \mathrm{CDCl}_{3}\right) \delta: 177.46,143.69,136.45,128.42,128.00$, 127.92, 122.44, 76.89, 72.16, 65.95, 49.82, 47.64, 46.76, 45.90, 41.85, 41.74, 41.41, 39.31, 38.14, 36.92, 33.88, 33.10, 32.54, 32.39, 30.71, 27.64, 26.84, 25.90, 23.65, 23.39, 23.07, 18.50, 16.92, 15.67, 11.37. MS (FAB) $\mathrm{m} / \mathrm{z}$ $585.2(\mathrm{M}+\mathrm{Na})^{+}$; HRMS (FAB). Calcd for $\mathrm{C}_{37} \mathrm{H}_{54} \mathrm{O}_{4} \mathrm{Na}$ : 585.3920. Found: 585.3912. (Figure $\mathrm{S4}$ ).

Oleanolic acid benzyl ester (PT5) [31,38]. Compound PT5 was obtained from OA and benzyl bromide as a white solid (185 mg, 68\%). ${ }^{1} \mathrm{H}$ NMR (600 MHz, $\left.\mathrm{CDCl}_{3}\right)$ 8: 7.35-7.29 (m, 5H, H-Ar), 5.29 $(\mathrm{t}, J=3.6 \mathrm{~Hz}, 1 \mathrm{H}), 5.09\left(\mathrm{~d}, J=12.6 \mathrm{~Hz}, 1 \mathrm{H}, \mathrm{Bn}-\mathrm{CH} H^{\prime}\right), 5.05\left(\mathrm{~d}, J=12.6 \mathrm{~Hz}, 1 \mathrm{H}, \mathrm{Bn}-\mathrm{CHH}^{\prime}\right), 3.20(\mathrm{dt}$, $J=10.1,4.9 \mathrm{~Hz}, 1 \mathrm{H}), 2.90(\mathrm{dd}, J=13.8,4.4 \mathrm{~Hz}, 1 \mathrm{H}), 1.98(\mathrm{td}, J=13.8,4.1 \mathrm{~Hz}, 1 \mathrm{H}), 1.85(\mathrm{dd}, J=8.9,3.7$ $\mathrm{Hz}, 2 \mathrm{H}), 1.74-1.59(\mathrm{~m}, 7 \mathrm{H}), 1.57-1.50(\mathrm{~m}, 4 \mathrm{H}), 1.42(\mathrm{td}, J=12.6,3.7 \mathrm{~Hz}, 1 \mathrm{H}), 1.37-1.16(\mathrm{~m}, 7 \mathrm{H}), 1.12(\mathrm{~s}$, $3 \mathrm{H}), 1.04(\mathrm{dt}, J=6.6,3.5 \mathrm{~Hz}, 1 \mathrm{H}), 0.98(\mathrm{~s}, 3 \mathrm{H}), 0.92(\mathrm{~s}, 3 \mathrm{H}), 0.90(\mathrm{~s}, 3 \mathrm{H}), 0.88(\mathrm{~s}, 3 \mathrm{H}), 0.77(\mathrm{~s}, 3 \mathrm{H}), 0.71(\mathrm{dd}$, $J=11.7,1.8 \mathrm{~Hz}, 1 \mathrm{H}), 0.61(\mathrm{~s}, 3 \mathrm{H}) .{ }^{13} \mathrm{C}$ NMR $\left(150 \mathrm{MHz}, \mathrm{CDCl}_{3}\right) \delta: 177.46,143.72,136.48,128.42,127.99$, $127.91,122.53,79.04,65.94,55.25,47.65,46.78,45.92,41.73,41.42,39.33,38.77,38.48,37.04,33.90,33.11$, $32.76,32.41,31.59,30.71,28.12,27.66,27.23,25.89,23.66,23.42,23.09,22.66,18.34,16.91,15.58,15.31$, 14.11. MS (FAB) $m / z$ 569.4 (M + Na) ${ }^{+}$; HRMS (FAB). Calcd for $\mathrm{C}_{37} \mathrm{H}_{54} \mathrm{O}_{3} \mathrm{Na}$ : 569.3967 . Found: 569.4046 . (Figure S5).

Ursolic acid benzyl ester (PT6) [38]. Compound PT6 was obtained from UA and benzyl bromide as a white solid $(221 \mathrm{mg}, 81 \%) .{ }^{1} \mathrm{H}$ NMR $\left(600 \mathrm{MHz}, \mathrm{CDCl}_{3}\right) 8: 7.36-7.30(\mathrm{~m}, 5 \mathrm{H}, \mathrm{H}-\mathrm{Ar}), 5.23(\mathrm{t}, J=3.6 \mathrm{~Hz}$, $1 \mathrm{H}), 5.10\left(\mathrm{~d}, J=12.5 \mathrm{~Hz}, 1 \mathrm{H}, \mathrm{Bn}-\mathrm{CH} H^{\prime}\right), 4.98\left(\mathrm{~d}, J=12.5 \mathrm{~Hz}, 1 \mathrm{H}, \mathrm{Bn}-\mathrm{CHH}^{\prime}\right), 3.21(\mathrm{dd}, J=11.3,4.7 \mathrm{~Hz}$, $1 \mathrm{H}), 2.26(\mathrm{dd}, J=11.3,0.9 \mathrm{~Hz}, 1 \mathrm{H}), 2.01(\mathrm{td}, J=13.4,4.5 \mathrm{~Hz}, 1 \mathrm{H}), 1.90-1.83(\mathrm{~m}, 2 \mathrm{H}), 1.82-1.76(\mathrm{~m}, 1 \mathrm{H})$, $1.73-1.70(\mathrm{~m}, 2 \mathrm{H}), 1.67-1.44(\mathrm{~m}, 9 \mathrm{H}), 1.36-1.25(\mathrm{~m}, 6 \mathrm{H}), 1.07(\mathrm{~s}, 3 \mathrm{H}), 1.05-1.01(\mathrm{~m}, 1 \mathrm{H}), 0.98(\mathrm{~s}, 3 \mathrm{H}), 0.93$ $(\mathrm{d}, J=6.4 \mathrm{~Hz}, 3 \mathrm{H}), 0.89(\mathrm{~s}, 3 \mathrm{H}), 0.85(\mathrm{~d}, J=6.4 \mathrm{~Hz}, 3 \mathrm{H}), 0.77(\mathrm{~s}, 3 \mathrm{H}), 0.70(\mathrm{dd}, J=11.7,1.3 \mathrm{~Hz}, 1 \mathrm{H}), 0.64$ (s, 3H). ${ }^{13} \mathrm{C} \mathrm{NMR}\left(150 \mathrm{MHz}, \mathrm{CDCl}_{3}\right) \delta: 177.29,138.13,136.41,128.41,128.16,127.95,125.74,79.04,65.99$, 55.26, 52.93, 48.15, 47.60, 42.07, 39.56, 39.12, 38.87, 38.77, 38.67, 36.98, 36.66, 33.07, 31.60, 30.70, 28.17, $27.99,27.26,24.28,23.59,23.29,22.66,21.19,18.33,17.04,17.01,15.64,15.46,14.13$. MS (FAB) $m / z 569.3$ $(\mathrm{M}+\mathrm{Na})^{+}$; HRMS (FAB). Calcd for $\mathrm{C}_{37} \mathrm{H}_{54} \mathrm{O}_{3} \mathrm{Na}$ : 569.3971. Found: 569.3970. (Figure S6).

Gypsogenin 4-methoxybenzyl ester (GP1). Compound GP1 was obtained from GP and 4-methoxybenzyl bromide as a white solid (123 mg, 42\%); mp 78-80 ${ }^{\circ} \mathrm{C} .{ }^{1} \mathrm{H}$ NMR $\left(600 \mathrm{MHz}, \mathrm{CDCl}_{3}\right)$

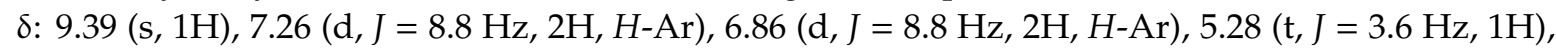
$5.02\left(\mathrm{~d}, J=12.1 \mathrm{~Hz}, 1 \mathrm{H}, \mathrm{Bn}-\mathrm{CH} H^{\prime}\right), 4.97\left(\mathrm{~d}, J=12.1 \mathrm{~Hz}, 1 \mathrm{H}, \mathrm{Bn}-\mathrm{CHH}^{\prime}\right), 3.80(\mathrm{~s}, 3 \mathrm{H}), 3.76(\mathrm{dd}, J=11.6$, $4.3 \mathrm{~Hz}, 1 \mathrm{H}), 2.88(\mathrm{dd}, J=13.9,4.3 \mathrm{~Hz}, 1 \mathrm{H}), 1.95(\mathrm{td}, J=13.6,4.1 \mathrm{~Hz}, 1 \mathrm{H}), 1.87(\mathrm{dd}, J=8.9,3.6 \mathrm{~Hz}, 2 \mathrm{H})$, $1.71-1.44(\mathrm{~m}, 14 \mathrm{H}), 1.32(\mathrm{td}, J=13.9,4.3 \mathrm{~Hz}, 1 \mathrm{H}), 1.27-1.25(\mathrm{~m}, 1 \mathrm{H}), 1.19-1.14(\mathrm{~m}, 2 \mathrm{H}), 1.13(\mathrm{~s}, 3 \mathrm{H})$, $1.06(\mathrm{~s}, 3 \mathrm{H}), 1.04-1.02(\mathrm{~m}, 1 \mathrm{H}), 1.04-0.97(\mathrm{~m}, 3 \mathrm{H}), 0.93(\mathrm{~s}, 3 \mathrm{H}), 0.91(\mathrm{~s}, 3 \mathrm{H}), 0.89(\mathrm{~s}, 3 \mathrm{H}), 0.59(\mathrm{~s}, 3 \mathrm{H}) .{ }^{13} \mathrm{C}$ NMR $\left(150 \mathrm{MHz}, \mathrm{CDCl}_{3}\right)$ ): 207.08, 177.41, 159.46, 143.84, 129.84, 128.59, 122.06, 113.81, 71.93, 65.75, 55.29, 55.19, 48.25, 47.56, 46.65, 45.91, 41.79, 41.42, 39.67, 38.07, 36.01, 33.87, 33.10, 32.31, 32.14, 30.71, $27.60,26.13,25.89,23.65,23.37,22.99,20.76,16.91,15.57,8.95$. MS (FAB) $m / z 613.5(\mathrm{M}+\mathrm{Na})^{+}$; HRMS (FAB). Calcd for $\mathrm{C}_{38} \mathrm{H}_{54} \mathrm{O}_{5} \mathrm{Na}$ : 613.3869. Found: 613.3865. (Figure S7).

Gypsogenin 3,5-bis(trifluromethyl)benzyl ester (GP2): Compound GP2 was obtained from GP and 3,5-bis(trifluoromethyl)benzyl bromide as a white solid (149 mg, 43\%); mp 85-87 ${ }^{\circ} \mathrm{C} .{ }^{1} \mathrm{H}$ NMR $\left(600 \mathrm{MHz}, \mathrm{CDCl}_{3}\right) \delta: 9.39$ (s, 1H), $7.83(\mathrm{~s}, 1 \mathrm{H}, \mathrm{H}-\mathrm{Ar}), 7.81(\mathrm{~s}, 2 \mathrm{H}, \mathrm{H}-\mathrm{Ar}), 5.28(\mathrm{t}, J=3.6 \mathrm{~Hz}, 1 \mathrm{H}), 5.20$ (d, $\left.J=13.2 \mathrm{~Hz}, 1 \mathrm{H}, \mathrm{Bn}-\mathrm{CHH}^{\prime}\right), 5.13\left(\mathrm{~d}, J=13.2 \mathrm{~Hz}, 1 \mathrm{H}, \mathrm{Bn}-\mathrm{CHH}^{\prime}\right), 3.76(\mathrm{dd}, J=11.5,4.5 \mathrm{~Hz}, 1 \mathrm{H}), 2.89$ $(\mathrm{dd}, J=13.7,4.1 \mathrm{~Hz}, 1 \mathrm{H}), 2.01(\mathrm{td}, J=13.7,4.1 \mathrm{~Hz}, 1 \mathrm{H}), 1.87-1.84(\mathrm{~m}, 2 \mathrm{H}), 1.74-1.44(\mathrm{~m}, 14 \mathrm{H}), 1.36(\mathrm{td}$, $J=13.7,4.1 \mathrm{~Hz}, 1 \mathrm{H}), 1.26-1.17(\mathrm{~m}, 3 \mathrm{H}), 1.15(\mathrm{~s}, 3 \mathrm{H}), 1.06(\mathrm{~s}, 3 \mathrm{H}), 0.98-0.95(\mathrm{~m}, 1 \mathrm{H}), 0.93(\mathrm{~s}, 3 \mathrm{H}), 0.91$ $(\mathrm{s}, 3 \mathrm{H}), 0.89(\mathrm{~s}, 3 \mathrm{H}), 0.48(\mathrm{~s}, 3 \mathrm{H}) .{ }^{13} \mathrm{C}$ NMR $\left(150 \mathrm{MHz}, \mathrm{CDCl}_{3}\right) \delta: 207.10,177.18,143.69,138.97,131.92$ $(\mathrm{q}, J=33.5 \mathrm{~Hz}), 128.09,124.09,122.32,122.01-121.91(\mathrm{~m}), 71.94,64.35,55.15,48.20,47.46,46.88,45.85$, $41.77,41.38$, 39.61, 38.01, 35.97, 33.78, 33.01, 32.39, 31.98, 31.58, 30.66, 27.49, 26.10, 25.89, 23.51, 23.19, 
23.11, 22.64, 20.65, 16.67, 15.36, 14.09, 8.95. MS (FAB) $m / z 719.4(\mathrm{M}+\mathrm{Na})^{+}$; HRMS (FAB). Calcd for $\mathrm{C}_{39} \mathrm{H}_{50} \mathrm{O}_{4} \mathrm{~F}_{6} \mathrm{Na}$ : 719.3511. Found: 719.3511. (Figure S8).

Gypsogenin 4-trifluoromethyl benzyl ester (GP3). Compound GP3 was obtained from GP and 4-trifluoromethyl benzyl bromide as a white solid (128 mg, 41\%); mp 175-177 ${ }^{\circ} \mathrm{C} .{ }^{1} \mathrm{H} \mathrm{NMR}(600 \mathrm{MHz}$, $\left.\mathrm{CDCl}_{3}\right) \delta: 9.39(\mathrm{~s}, 1 \mathrm{H}), 7.60(\mathrm{~d}, J=8.0 \mathrm{~Hz}, 2 \mathrm{H}, \mathrm{H}-\mathrm{Ar}), 7.46(\mathrm{~d}, J=8.0 \mathrm{~Hz}, 2 \mathrm{H}, \mathrm{H}-\mathrm{Ar}), 5.28(\mathrm{t}, J=3.6 \mathrm{~Hz}$, $1 \mathrm{H}), 5.15\left(\mathrm{~d}, J=12.9 \mathrm{~Hz}, 1 \mathrm{H}, \mathrm{Bn}-\mathrm{CH} H^{\prime}\right), 5.07\left(\mathrm{~d}, J=12.9 \mathrm{~Hz}, 1 \mathrm{H}, \mathrm{Bn}-\mathrm{CHH}^{\prime}\right), 3.76(\mathrm{dd}, J=11.4,4.4 \mathrm{~Hz}$, $1 \mathrm{H}), 2.89(\mathrm{dd}, J=13.5,4.1 \mathrm{~Hz}, 1 \mathrm{H}), 1.99(\mathrm{td}, J=13.5,4.1 \mathrm{~Hz}, 1 \mathrm{H}), 1.87-1.84(\mathrm{~m}, 2 \mathrm{H}), 1.74-1.19(\mathrm{~m}, 7 \mathrm{H})$, $1.55(\mathrm{ddd}, J=18.5,11.1,4.1 \mathrm{~Hz}, 2 \mathrm{H}), 1.49-1.42(\mathrm{~m}, 3 \mathrm{H}), 1.34(\mathrm{td}, J=13.7,4.1 \mathrm{~Hz}, 1 \mathrm{H}), 1.21(\mathrm{ddd}, J=13.5$, 4.1, $2.1 \mathrm{~Hz}, 2 \mathrm{H}), 1.14(\mathrm{~s}, 3 \mathrm{H}), 1.06(\mathrm{~s}, 3 \mathrm{H}), 1.04-0.96(\mathrm{~m}, 3 \mathrm{H}), 0.93(\mathrm{~s}, 3 \mathrm{H}), 0.91(\mathrm{~s}, 6 \mathrm{H}), 0.89-0.87(\mathrm{~m}, 1 \mathrm{H})$, $0.86-0.83(\mathrm{~m}, 1 \mathrm{H}), 0.50(\mathrm{~s}, 3 \mathrm{H}) .{ }^{13} \mathrm{C}$ NMR $\left(150 \mathrm{MHz}, \mathrm{CDCl}_{3}\right) \delta: 207.09,177.22,143.73,140.35,128.27$, $125.42(\mathrm{q}, J=3.8 \mathrm{~Hz}), 123.15,122.22,71.95,65.07,55.16,48.24,47.50,46.78,45.86,41.78,41.45,39.64$, 38.06, 35.99, 33.82, 33.06, 32.38, 32.10, 30.70, 29.71, 27.57, 26.12, 25.90, 23.62, 23.35, 23.06, 20.67, 16.77, 15.49, 8.95. MS (FAB) $m / z 651.5(\mathrm{M}+\mathrm{Na})^{+}$; HRMS (FAB). Calcd for $\mathrm{C}_{38} \mathrm{H}_{51} \mathrm{O}_{4} \mathrm{~F}_{3} \mathrm{Na}$ : 651.3637. Found: 651.3605. (Figure S9).

Gypsogenin 3,5-difluorobenzyl ester (GP4). Compound GP4 was obtained from GP and 3,5-difluorobenzyl bromide as a white solid (119 mg, 40\%); mp 58-60 ${ }^{\circ} \mathrm{C} .{ }^{1} \mathrm{H}$ NMR $(600 \mathrm{MHz}$, $\left.\mathrm{CDCl}_{3}\right) \delta: 9.40(\mathrm{~s}, 1 \mathrm{H}), 6.86(\mathrm{dd}, J=7.9,1.8 \mathrm{~Hz}, 2 \mathrm{H}, H-\mathrm{Ar}), 6.76-6.72(\mathrm{~m}, 1 \mathrm{H}, H-\mathrm{Ar}), 5.32(\mathrm{t}, J=3.6 \mathrm{~Hz}$, $1 \mathrm{H}), 5.09\left(\mathrm{~d}, J=13.1 \mathrm{~Hz}, 1 \mathrm{H}, \mathrm{Bn}-\mathrm{CH} H^{\prime}\right), 4.96\left(\mathrm{~d}, J=13.1 \mathrm{~Hz}, 1 \mathrm{H}, \mathrm{Bn}-\mathrm{CHH}^{\prime}\right), 3.77(\mathrm{~d}, J=10.0 \mathrm{~Hz}, 1 \mathrm{H}), 2.90$ $(\mathrm{dd}, J=13.5,4.1 \mathrm{~Hz}, 1 \mathrm{H}), 2.00(\mathrm{td}, J=13.7,4.1 \mathrm{~Hz}, 1 \mathrm{H}), 1.89(\mathrm{dd}, J=8.9,3.6 \mathrm{~Hz}, 2 \mathrm{H}), 1.72-1.61(\mathrm{~m}, 7 \mathrm{H})$, $1.59(\mathrm{~s}, 3 \mathrm{H}), 1.56-1.44(\mathrm{~m}, 4 \mathrm{H}), 1.38-1.18(\mathrm{~m}, 6 \mathrm{H}), 1.15(\mathrm{~s}, 3 \mathrm{H}), 1.06(\mathrm{~s}, 3 \mathrm{H}), 1.05-1.04(\mathrm{~m}, 1 \mathrm{H}), 1.00-0.97$ $(\mathrm{m}, 1 \mathrm{H}), 0.93(\mathrm{~s}, 3 \mathrm{H}), 0.91(\mathrm{~s}, 3 \mathrm{H}), 0.88(\mathrm{t}, J=7.0 \mathrm{~Hz}, 1 \mathrm{H}), 0.59(\mathrm{~s}, 3 \mathrm{H}) .{ }^{13} \mathrm{C} \mathrm{NMR}\left(150 \mathrm{MHz}, \mathrm{CDCl}_{3}\right) \delta$ : 207.09, 177.16, 163.86 (d, $J=12.5 \mathrm{~Hz}), 162.21$ (d, $J=12.5 \mathrm{~Hz}), 143.72,140.26,122.35,110.55,110.42$, $103.29,71.94,64.55,55.49,55.17,48.26,47.51,46.83,45.86,41.81,41.45,39.68,38.05,36.02,33.81,33.06$, $32.39,32.14,31.59,30.70,27.56,26.12,25.91,23.61,23.30,23.07,22.65,20.74,16.85,15.53,14.11,8.96$. MS (FAB) $m / z 619.2(\mathrm{M}+\mathrm{Na})^{+}$; HRMS (FAB). Calcd for $\mathrm{C}_{37} \mathrm{H}_{50} \mathrm{O}_{4} \mathrm{~F}_{2} \mathrm{Na}$ : 619.3575. Found: 619.3576. (Figure S10).

Gypsogenin 4-cyanobenzyl ester (GP5). Compound GP5 was obtained from GP and 4-cyanobenzyl bromide as a white solid $(140 \mathrm{mg}, 48 \%) ; \mathrm{mp} 175-177^{\circ} \mathrm{C} .{ }^{1} \mathrm{H}$ NMR $\left(600 \mathrm{MHz}, \mathrm{CDCl}_{3}\right) \delta 9.39(\mathrm{~s}, 1 \mathrm{H}), 7.65$ $(\mathrm{d}, J=8.3 \mathrm{~Hz}, 2 \mathrm{H}, H-\mathrm{Ar}), 7.45(\mathrm{~d}, J=8.3 \mathrm{~Hz}, 2 \mathrm{H}, H-\mathrm{Ar}), 5.29(\mathrm{t}, J=3.5 \mathrm{~Hz}, 1 \mathrm{H}), 5.16(\mathrm{~d}, J=13.3 \mathrm{~Hz}, 1 \mathrm{H}$, Bn-CHH'), $5.05\left(\mathrm{~d}, J=13.3 \mathrm{~Hz}, 1 \mathrm{H}, \mathrm{Bn}-\mathrm{CHH}^{\prime}\right), 3.76(\mathrm{dd}, J=11.4,4.1 \mathrm{~Hz}, 1 \mathrm{H}), 2.89(\mathrm{dd}, J=13.8,4.1 \mathrm{~Hz}$, $1 \mathrm{H}), 2.00(\mathrm{td}, J=13.7,4.1 \mathrm{~Hz}, 1 \mathrm{H}), 1.90-1.77(\mathrm{~m}, 2 \mathrm{H}), 1.73-1.45(\mathrm{~m}, 12 \mathrm{H}), 1.35(\mathrm{td}, J=13.7,4.1 \mathrm{~Hz}, 1 \mathrm{H})$, 1.27-1.17 (m, 4H), $1.15(\mathrm{~s}, 3 \mathrm{H}), 1.06(\mathrm{~s}, 3 \mathrm{H}), 1.05-1.04(\mathrm{~m}, 1 \mathrm{H}), 1.00-0.97(\mathrm{~m}, 1 \mathrm{H}), 0.93(\mathrm{~s}, 3 \mathrm{H}), 0.92(\mathrm{~s}, 3 \mathrm{H})$, $0.91(\mathrm{~s}, 3 \mathrm{H}), 0.88(\mathrm{t}, J=7.0 \mathrm{~Hz}, 1 \mathrm{H}), 0.56(\mathrm{~s}, 3 \mathrm{H}) .{ }^{13} \mathrm{C} \mathrm{NMR}\left(150 \mathrm{MHz}, \mathrm{CDCl}_{3}\right) \delta 207.12,177.16,143.67$, 141.70, 132.30, 128.37, 122.27, 118.56, 111.87, 71.95, 64.86, 55.17, 48.20, 47.48, 46.85, 46.84, 45.81, 41.80, $41.79,41.47,39.66,38.05,35.99,33.79,33.04,32.38,32.11,31.58,30.70,30.69,30.68,30.68,27.57,26.11$, $25.90,23.61,23.35,23.06,22.65,20.71,16.87,15.57,14.11,8.98$. MS (FAB) $m / z 608.5(\mathrm{M}+\mathrm{Na})^{+}$; HRMS (FAB). Calcd for $\mathrm{C}_{38} \mathrm{H}_{51} \mathrm{NO}_{4} \mathrm{Na:}$ 608.3716. Found: 608.3710. (Figure S11).

\subsection{Biochemistry}

\subsubsection{Cell Cultures}

A549 human lung carcinoma and HeLa human cervical carcinoma cell lines were cultured in Dulbecco's Modified Eagle Medium (DMEM). MCF7 human breast cancer and leukemic (K562, HL-60, MT-2 and Jurkat) cells, and PBMC (Precision Bioservices, Frederic, MD, USA) were cultured in RPMI 1640. All media were purchased from FUJIFILM Wako (Osaka, Japan) and supplemented with $10 \%$ fetal bovine serum (FBS) (Sigma-Aldrich, St. Louis, MO, USA) and $89 \mu \mathrm{g} / \mathrm{mL}$ streptomycin (Meiji Seika Pharma, Tokyo, Japan) at $37{ }^{\circ} \mathrm{C}$ in a humid atmosphere and $5 \% \mathrm{CO}_{2}$. In experiments, all cancer cell lines and PBMC were cultured in 24-well and 96-well plates (Iwaki brand Asahi Glass, Chiba, Japan) at $2 \times 10^{4}$ cells $/ \mathrm{mL}$ and $1 \times 10^{6}$ cells $/ \mathrm{mL}$ concentrations, respectively for $24 \mathrm{~h}$. The stock solution of compounds and imatinib in concentrations between 0,1-10 $\mathrm{mM}$ were prepared in DMSO (FUJIFILM 
Wako, Osaka, Japan) and further diluted with fresh culture medium. The concentration of DMSO in the final culture medium was $1 \%$, which had no effect on the cell viability [40].

\subsubsection{Cell Viability Assay}

The effect of PTs, their derivatives and imatinib on cell viability was assessed by using MTT (Dojindo, Kumamoto, Japan) as previously described in the literature [41,42]. The cells were exposed to various concentrations $(1-100 \mu \mathrm{M})$ of the compounds for $24 \mathrm{~h}$ at $37^{\circ} \mathrm{C}$. The cells were then stained with MTT solution and incubated for additional $4 \mathrm{~h}$. At the end of this period, supernatants were removed and $100 \mu \mathrm{L}$ DMSO was added to each well to solubilize the formazan crystals. The absorbance of the solution was determined on the Infinitive M1000 (Tecan, Mannedorf, Switzerland) plate reader at a wavelength of $550 \mathrm{~nm}$ with background subtraction at $630 \mathrm{~nm}$. All experiments were performed in triplicate and $\mathrm{IC}_{50}$ values defined as the drug concentrations that reduced absorbance to $50 \%$ of control values were calculated from MTT results.

\subsubsection{Cell Death Detection}

After the cells were incubated with the selected compounds in this series at $\mathrm{IC}_{50}$ concentration for $6 \mathrm{~h}$, the apoptotic/necrotic/healthy/detection kit (PromoKine, Heidelberg, Germany) was performed according to the manufacturer's instructions. The cells were then briefly washed twice with $1 \times$ binding buffer. A staining solution containing $50 \mu \mathrm{L}$ of $1 \times$ binding buffer and $5 \mu \mathrm{L}$ of FITC-Annexin V solution, $5 \mu \mathrm{L}$ of ethidium homodimer III solution, or $5 \mu \mathrm{L}$ of Hoechst 33342 solution was added, and incubated for $15 \mathrm{~min}$ at room temperature in the dark. Then, the cells were washed with $1 \times$ binding buffer, and analyzed by the all-in-one fluorescence microscope Biorevo Fluorescence BZ-9000 (Keyence, Osaka, Japan). The number of healthy cells (Hoechst 33342), apoptotic cells (Annexin V), late apoptotic or necrotic cells (Annexin V and Ethidium homodimer III) and necrotic cells (Ethidium homodimer III) were counted as previously described [43].

\subsubsection{Determination of ABL1 Kinase Inhibition}

The ABL1 kinase profiling assay protocol was applied according to the manufacturer's instructions (Promega, Madison, WI, USA) with the modification proposed in [36]. In this system, the ABL1 kinase and its substrate were diluted with $95 \mu \mathrm{L} 2.5 \mathrm{x}$ Kinase Buffer and $15 \mu \mathrm{L}$ of $100 \mu \mathrm{M}$ ATP solutions, respectively. Then, the reaction of ABL1 kinase was performed using $2 \mu \mathrm{L}$ of the compound solution at varying concentrations $(0.1-30 \mu \mathrm{M}), 4 \mu \mathrm{L}$ of kinase working stock, and $4 \mu \mathrm{L}$ of $\mathrm{ATP} /$ substrate working stock in the 384-well plate. After $1 \mathrm{~h}$ of incubation at room temperature, the activity of the ABL1 kinase was detected using the ADP-Glo Kinase Assay (Promega, Madison, WI, USA) and the inhibitory kinase activity of compounds in dose-response mode was measured by the Infinitive M1000 luminescence plate reader (Tecan, Groding, Austria). The $\mathrm{IC}_{50}$ values of tested compounds required to decrease the kinase activity by $50 \%$ were calculated.

\subsubsection{Immunoblot Analysis}

The K562 cells were cultured with compounds GP2, GP5, PT5 and imatinib at $10 \mu \mathrm{M}$ concentrations. After $6 \mathrm{~h}$ of treatment at $37^{\circ} \mathrm{C}$, the cells were lysed in PBS-Laemmli sample buffer and then, immunoblot analysis was conducted using phospho-specific-p44/42 MAPK (Erk1/2) (Thr202/Tyr204) (D13.14.4E) XP Rabbit mAb (1:1000) (Cell Signaling Technology, Danvers, MA, USA) or anti- $\beta$-actin clone AC-15 (Sigma-Aldrich, St. Louis, MO, USA). For immunoreactivity detection, the chemiluminescence method was performed as previously described $[44,45]$. The band intensity was measured using the ImageJ software (NIH, Bethesda, MD, USA). 


\subsection{Molecular Docking}

Crystal structure of the ABL kinase in complex with imatinib was obtained from Protein Data Bank (PDB: 1IEP); PT5 was built by ChemDraw Professional 16. Before the docking simulations, PT5 and 1IEP preparation included the addition of hydrogens, the assignment of bond order, and assessment of the correct protonation as previously described [46,47]. The MOE 2019.01 software (Chemical Computing Group, Montreal, Canada) was employed for the preparation, interactive docking, visualization and the analysis procedures using its default parameters [48-50].

\section{Conclusions}

We herein designed and synthesized benzyl esters of different triterpenes (PT1-PT6) and the newly substituted benzyl esters of gypsogenin (GP1-GP5), based on our finding that 1c (benzyl ester of gypsogenin) has anti-ABL kinase and anti-CML activities [36]. These derivatives and the parent triterpenes were biologically evaluated, and three compounds with good activities, GP2, GP5 and PT5, were found. Among them, PT5 has six times stronger in vitro $\mathrm{IC}_{50}$ against ABL kinase compared to $1 c$, and stronger activity against CML cell line K562. The stronger interaction of PT5 with ABL kinase was explained using the difference between the molecular docking by the methyl group in PT5 on its C4 position and the aldehyde group on the same position of 1c. The PT5 was shown to inhibit ERK signaling to induce apoptosis in K562. The other compounds GP2 and GP5 have stronger activity against K562 than PT5, although their in vitro activity against ABL1 is weaker. It is considered that these two compounds have different mechanisms to induce cell death from inhibition of BCR-ABL. Actually, GP2 and GP5 have stronger activity against some cell lines other than K562. Taken together, we succeeded in increasing the activity against ABL kinase and CML cells by derivatization. The strongest inhibitor PT5 is based on oleanolic acid which is widely spread in many plants. This study would lead to the basis of new anti-CML drugs based on naturally found pentacyclic triterpens.

Supplementary Materials: The following are available online. Figure S1: ${ }^{1} \mathrm{H}$ and ${ }^{13} \mathrm{C}$ spectra of PT1; Figure S2: ${ }^{1} \mathrm{H}$ and ${ }^{13} \mathrm{C}$ spectra of PT2; Figure S3: ${ }^{1} \mathrm{H}$ and ${ }^{13} \mathrm{C}$ spectra of PT3; Figure S4: ${ }^{1} \mathrm{H}$ and ${ }^{13} \mathrm{C}$ spectra of PT4; Figure S5: ${ }^{1} \mathrm{H}$ and ${ }^{13} \mathrm{C}$ spectra of PT5; Figure S6: ${ }^{1} \mathrm{H}$ and ${ }^{13} \mathrm{C}$ spectra of PT6; Figure S7: ${ }^{1} \mathrm{H}$ and ${ }^{13} \mathrm{C}$ spectra of GP1; Figure S8: ${ }^{1} \mathrm{H}$ and ${ }^{13} \mathrm{C}$ spectra of GP2; Figure S9: ${ }^{1} \mathrm{H}$ and ${ }^{13} \mathrm{C}$ spectra of GP3; Figure S10: ${ }^{1} \mathrm{H}$ and ${ }^{13} \mathrm{C}$ spectra of GP4; Figure S11: ${ }^{1} \mathrm{H}$ and ${ }^{13} \mathrm{C}$ spectra of GP5.

Author Contributions: H.I.C., M.O.R. and M.F. designed the research; S.E.O., N.G.U. and E.S. performed isolation of gypsogenin; M.O.R. synthesized and purified the compounds and H.I.A.-A. interpreted spectroscopic data. H.I.C. was responsible for the biological work and mainly wrote the manuscript. D.E.E. and H.T. performed a part of biological experiment. M.O.R. undertook the docking study. Z.O. took part in the analysis of biological data and discussion of the results. M.C., T.F.S.A. and N.Y. did discussion. M.F. and M.O. were responsible for the correspondence of the manuscript. All authors discussed, edited and approved the final version.

Funding: This study was supported by Bilateral Joint Research Project from the Japanese Society for the Promotion of Science (the grant no: 18039111-000102).

Acknowledgments: MCF7 and A549 cells were provided by the RIKEN BRC through the National Bio Resource Project of the MEXT/AMED, Japan.

Conflicts of Interest: The authors report no conflicts of interest.

\section{References}

1. Fitzmaurice, C.; Akinyemiju, T.F.; Al Lami, F.H.; Alam, T.; Alizadeh-Navaei, R.; Allen, C.; Alsharif, U.; Alvis-Guzman, N.; Amini, E.; Anderson, B.O.; et al. Global, Regional, and National Cancer Incidence, Mortality, Years of Life Lost, Years Lived With Disability, and Disability-Adjusted Life-Years for 29 Cancer Groups, 1990 to 2016 A Systematic Analysis for the Global Burden of Disease Study. Jama Oncol. 2018, 4, 1553-1568. [PubMed]

2. Szczepański, T.; van der Velden, V.H.; van Dongen, J.J. Classification systems for acute and chronic leukaemias. Best Pract. Res. Clin. Haematol. 2013, 16, 561-582. [CrossRef] 
3. Clarkson, B.; Strife, A.; Wisniewski, D.; Lambek, C.L.; Liu, C. Chronic myelogenous leukemia as a paradigm of early cancer and possible curative strategies. Leukemia 2003, 17, 1211-1262. [CrossRef] [PubMed]

4. $\quad$ Kang, Z.J.; Liu, Y.F.; Xu, L.Z.; Long, Z.J.; Huang, D.; Yang, Y.; Liu, B.; Feng, J.X.; Pan, Y.J.; Yan, J.S.; et al. The Philadelphia chromosome in leukemogenesis. Chin. J. Cancer 2016, 35. [CrossRef] [PubMed]

5. Ernst, T.; Obstfelder, E.; Hochhaus, A. Chronic myeloid leukemia. Onkologe 2018, 24, 427-441. [CrossRef]

6. Schiffer, C.A. Diagnosis and treatment of chronic myeloid leukemia. In Neoplastic Diseases of the Blood, 6th ed.; Wiernik, P.H., Dutcher, J.P., Gertz, M.A., Eds.; Springer: New York, NY, USA, 2018; Volume 20, p. 64.

7. De Braekeleer, E.; Douet-Guilbert, N.; Rowe, D.; Bown, N.; Morel, F.; Berthou, C.; Ferec, C.; De Braekeleer, M. ABL1 fusion genes in hematological malignancies: A review. Eur. J. Haematol. 2011, 86, 361-371. [CrossRef]

8. Iqbal, Z. A comprehensive analysis of breakpoint cluster region-abelson fusion oncogene splice variants in chronic myeloid leukemia and their correlation with disease biology. Indian, J. Hum. Genet. 2014, 20, 64-68. [CrossRef]

9. Ross, T.S.; Mgbemena, V.E. Re-evaluating the role of BCR/ABL in chronic myelogenous leukemia. Mol. Cell Oncol. 2014, 1, e963450. [CrossRef]

10. Vlahovic, G.; Crawford, J. Activation of tyrosine kinases in cancer. Oncologist 2003, 8, 531-538. [CrossRef]

11. Lemmon, M.A.; Schlessinger, J. Cell Signaling by Receptor Tyrosine Kinases. Cell 2010, 141, 1117-1134. [CrossRef]

12. Du, Z.F.; Lovly, C.M. Mechanisms of receptor tyrosine kinase activation in cancer. Mol Cancer $2018,17$. [CrossRef] [PubMed]

13. Iqbal, N.; Iqbal, N. Imatinib: A breakthrough of targeted therapy in cancer. Chemother. Res. Prac. 2014, 2014. [CrossRef] [PubMed]

14. Sacha, T. Imatinib in chronic myeloid leukemia: An overview. Mediterr. J. Hematol. Infect. Dis. 2014, 6, e2014007. [CrossRef] [PubMed]

15. Pophali, P.A.; Patnaik, M.M. The Role of New Tyrosine Kinase Inhibitors in Chronic Myeloid Leukemia. Cancer J. 2016, 22, 40-50. [CrossRef] [PubMed]

16. Garcia-Gutierrez, J.V.; Herrera, P.; Abalo, L.L.; Rey, M.D.; Calbacho, M.; Ramos, L.; Ramos, P.; Montalban, C.; Lopez-Jimenez, J. Impact of Second-Generation Tyrosine Kinase Inhibitors As Second Line Treatment for Patients with Chronic Myeloid Leukemia. Blood 2011, 118, 1615.

17. Wehrle, J.; Pahl, H.L.; von Bubnoff, N. Ponatinib: A Third-Generation Inhibitor for the Treatment of CML. Recent Results Canc. 2014, 201, 99-107.

18. Jabbour, E.; Kantarjian, H.; Cortes, J. Use of Second- and Third-Generation Tyrosine Kinase Inhibitors in the Treatment of Chronic Myeloid Leukemia: An Evolving Treatment Paradigm. Cl. Lymph. Myelom. Leuk. 2015, 15, 323-334. [CrossRef]

19. Rossari, F.; Minutolo, F.; Orciuolo, E. Past, present, and future of Bcr-Abl inhibitors: From chemical development to clinical efficacy. J. Hematol. Oncol. 2018, 11. [CrossRef]

20. Cragg, G.M.; Newman, D.J. Natural products: A continuing source of novel drug leads. Bba-Gen. Subjects 2013, 1830, 3670-3695. [CrossRef]

21. Nasr, H.; Abdel-Aziz, M.S.; Radwan, M.O.; Shaaban, M. Bioactive Secondary Metabolites from Terrestrial Streptomyces baarnensis MH4. Brit. J. Pharm. Res. 2015, 5, 72-81. [CrossRef]

22. Bishayee, A.; Sethi, G. Bioactive natural products in cancer prevention and therapy: Progress and promise. Semin. Cancer Biol. 2016, 40-41, 1-3. [CrossRef] [PubMed]

23. Liu, Q.; Meng, X.; Li, Y.; Zhao, C.N.; Tang, G.Y.; Li, H.B. Antibacterial and Antifungal Activities of Spices. Int. J. Mol. Sci. 2017, 18, 1283. [CrossRef]

24. Aung, T.N.; Qu, Z.P.; Kortschak, R.D.; Adelson, D.L. Understanding the Effectiveness of Natural Compound Mixtures in Cancer through Their Molecular Mode of Action. Int. J. Mol. Sci. 2017, 18, 656. [CrossRef] [PubMed]

25. Ibrahim, M.A.; El-Alfy, A.T.; Ezel, K.; Radwan, M.O.; Shilabin, A.G.; Kochanowska-Karamyan, A.J.; Abd-Alla, H.I.; Otsuka, M.; Hamann, M.T. Marine Inspired 2-(5-Halo-1H-indol-3-yl)-N, N-dimethylethanamines as Modulators of Serotonin Receptors: An Example Illustrating the Power of Bromine as Part of the Uniquely Marine Chemical Space. Mar. Drugs 2017, 15, 248. [CrossRef]

26. Scharenberg, F.; Zidorn, C. Genuine and Sequestered Natural Products from the Genus Orobanche (Orobanchaceae, Lamiales). Molecules 2018, 23, 2821. [CrossRef] 
27. Dzubak, P.; Hajduch, M.; Vydra, D.; Hustova, A.; Kvasnica, M.; Biedermann, D.; Markova, L.; Urban, M.; Sarek, J. Pharmacological activities of natural triterpenoids and their therapeutic implications. Nat. Prod. Rep. 2006, 23, 394-411. [CrossRef] [PubMed]

28. Laszczyk, M.N. Pentacyclic Triterpenes of the Lupane, Oleanane and Ursane Group as Tools in Cancer Therapy. Planta. Med. 2009, 75, 1549-1560. [CrossRef]

29. Lu, Z.Z.; Jin, Y.L.; Qiu, L.; Lai, Y.R.; Pan, J.X. Celastrol, a novel HSP90 inhibitor, depletes Bcr-Abl and induces apoptosis in imatinib-resistant chronic myelogenous leukemia cells harboring T315I mutation. Cancer Lett. 2010, 290, 182-191. [CrossRef]

30. Radwan, M.O.; Ismail, M.A.H.; El-Mekkawy, S.; Ismail, N.S.M.; Hanna, A.G. Synthesis and biological activity of new 18 beta-glycyrrhetinic acid derivatives. Arab. J. Chem. 2016, 9, 390-399. [CrossRef]

31. Wang, R.; Zheng, Q.X.; Wang, W.; Feng, L.; Li, H.J.; Huai, Q.Y. Design and Synthesis of New Anticancer Glycyrrhetinic Acids and Oleanolic Acids. Biol. Pharm. Bull. 2017, 40, 703-710. [CrossRef]

32. Ciftci, H. Effects of glycyrrhetic acid on human chronic myelogenous leukemia cells. Turk. J. Pharm. Sci. in press. [CrossRef]

33. Ozturk, S.E.; Babahan, I.; Ozmen, A. Synthesis, characterization and in vitro anti-neoplastic activity of gypsogenin derivatives. Bioorg. Chem. 2014, 53, 15-23. [CrossRef] [PubMed]

34. Emirdag-Ozturk, S.; Karayildirim, T.; Capaci-Karagoz, A.; Alankus-Caliskan, O.; Ozmen, A.; Poyrazoglu-Coban, E. Synthesis, antimicrobial and cytotoxic activities, and structure-activity relationships of gypsogenin derivatives against human cancer cells. Eur. J. Med. Chem. 2014, 82, 565-573. [CrossRef] [PubMed]

35. Zhang, H.; Mu, Y.; Wang, F.; Song, L.; Sun, J.; Liu, Y.; Sun, J. Synthesis of gypsogenin derivatives with capabilities to arrest cell cycle and induce apoptosis in human cancer cells. R Soc. Open Sci. 2018, 5, 171510. [CrossRef] [PubMed]

36. Ciftci, H.I.; Ozturk, S.E.; Ali, T.F.S.; Radwan, M.O.; Tateishi, H.; Koga, R.; Ocak, Z.; Can, M.; Otsuka, M.; Fujita, M. The First Pentacyclic Triterpenoid Gypsogenin Derivative Exhibiting Anti-ABL1 Kinase and Anti-chronic Myelogenous Leukemia Activities. Biol. Pharm. Bull. 2018, 41, 570-574. [CrossRef] [PubMed]

37. Zhang, L.; Chen, J.; Gong, Y.; Liu, J.; Zhang, L.; Hua, W.; Sun, H. Synthesis and Biological Evaluation of Asiatic Acid Derivatives as Inhibitors of Glycogen Phosphorylases. Chem. Biodivers. 2009, 6, 864-874. [CrossRef] [PubMed]

38. Wolfram, R.K.; Heller, L.; Csuk, R. Targeting Mitochondria: Esters of Rhodamine B with Triterpenoids Are Mitocanic Triggers of Apoptosis. Eur. J. Med. Chem. 2018, 152, 21-30. [CrossRef] [PubMed]

39. Rodríguez-Hernández, D.; Demuner, A.J.; Barbosa, L.C.A.; Csuk, R.; Heller, L. Hederagenin as a Triterpene Template for the Development of New Antitumor Compounds. Eur. J. Med. Chem. 2015, 105, 57-62. [CrossRef]

40. Bayrak, N.; Yildirim, H.; Tuyun, A.F.; Kara, E.M.; Celik, B.O.; Gupta, G.K.; Ciftci, H.I.; Fujita, M.; Otsuka, M.; Nasiri, H.R. Synthesis, Computational Study, and Evaluation of In Vitro Antimicrobial, Antibiofilm, and Anticancer Activities of New Sulfanyl Aminonaphthoquinone Derivatives. Lett Drug Des. Discov. 2017, 14, 647-661. [CrossRef]

41. Ali, T.F.S.; Iwamaru, K.; Ciftci, H.I.; Koga, R.; Matsumoto, M.; Oba, Y.; Kurosaki, H.; Fujita, M.; Okamoto, Y.; Umezawa, K.; et al. Novel metal chelating molecules with anticancer activity. Striking effect of the imidazole substitution of the histidine-pyridine-histidine system. Bioorgan Med. Chem. 2015, 23, 5476-5482. [CrossRef]

42. Tateishi, H.; Monde, K.; Anraku, K.; Koga, R.; Hayashi, Y.; Ciftci, H.I.; DeMirci, H.; Higashi, T.; Motoyama, K.; Arima, H.; et al. A clue to unprecedented strategy to HIV eradication: "Lock-in and apoptosis". Sci. Rep-Uk 2017, 7, 8957. [CrossRef] [PubMed]

43. Altintop, M.D.; Ciftci, H.I.; Radwan, M.O.; Sever, B.; Kaplancikli, Z.A.; Ali, T.F.S.; Koga, R.; Fujita, M.; Otsuka, M.; Ozdemir, A. Design, Synthesis, and Biological Evaluation of Novel 1,3,4-Thiadiazole Derivatives as Potential Antitumor Agents against Chronic Myelogenous Leukemia: Striking Effect of Nitrothiazole Moiety. Molecules 2018, 23, 59. [CrossRef] [PubMed]

44. Ciftci, H.I.; Fujino, H.; Koga, R.; Yamamoto, M.; Kawamura, S.; Tateishi, H.; Iwatani, Y.; Otsuka, M.; Fujita, M. Mutational analysis of HIV-2 Vpx shows that proline residue 109 in the poly-proline motif regulates degradation of SAMHD1. Febs. Lett. 2015, 589, 1505-1514. [CrossRef] [PubMed] 
45. Koga, R.; Radwan, M.O.; Ejima, T.; Kanemaru, Y.; Tateishi, H.; Ali, T.F.S.; Ciftci, H.I.; Shibata, Y.; Taguchi, Y.; Inoue, J.; et al. A Dithiol Compound Binds to the Zinc Finger Protein TRAF6 and Suppresses Its Ubiquitination. ChemMedChem 2017, 12, 1935-1941. [CrossRef] [PubMed]

46. Tanaka, A.; Radwan, M.O.; Hamasaki, A.; Ejima, A.; Obata, E.; Koga, R.; Tateishi, H.; Okamoto, Y.; Fujita, M.; Nakao, M.; et al. A novel inhibitor of farnesyltransferase with a zinc site recognition moiety and a farnesyl group. Bioorg. Med. Chem. Lett. 2017, 27, 3862-3866. [CrossRef] [PubMed]

47. Radwan, M.O.; Koga, R.; Hida, T.; Ejima, T.; Kanemaru, Y.; Tateishi, H.; Okamoto, Y.; Inoue, J.; Fujita, M.; Otsuka, M. Minimum structural requirements for inhibitors of the zinc finger protein TRAF6. Bioorg. Med Chem. Lett. 2019, 29, 2162-2167. [CrossRef] [PubMed]

48. Nishimura, N.; Radwan, M.O.; Amano, M.; Endo, S.; Fujii, E.; Hayashi, H.; Ueno, S.; Ueno, N.; Tatetsu, H.; Hata, H.; et al. Novel p97/VCP inhibitor induces endoplasmic reticulum stress and apoptosis in both bortezomib-sensitive and-resistant multiple myeloma cells. Cancer Sci. 2019, 00, 1-13. [CrossRef] [PubMed]

49. El-Shaheny, R.; Fuchigami, T.; Yoshida, S.; Radwan, M.O.; Nakayama, M. Complementary HPLC, in Silico Toxicity, and Molecular Docking Studies for Investigation of the Potential Influences of Gastric Acidity and Nitrite Content on Paracetamol Safety. Microchem. J. 2019, 150, 104107. [CrossRef]

50. Sever, B.; Altıntop, D.M.; Radwan, M.O.; Özdemir, A.; Otsuka, M.; Fujita, M.; Ciftci, H.I. Design, synthesis and biological evaluation of a new series of thiazolyl-pyrazolines as dual EGFR and HER2 inhibitors. Eur. J. Med. Chem. 2019, 182, 111648. [CrossRef]

Sample Availability: Samples of the compounds (1c, PT1, PT2, PT3, PT4, PT5, PT6, GP1, GP2, GP3, GP4, GP5 and GP6) are available from the authors.

(C) 2019 by the authors. Licensee MDPI, Basel, Switzerland. This article is an open access article distributed under the terms and conditions of the Creative Commons Attribution (CC BY) license (http://creativecommons.org/licenses/by/4.0/). 